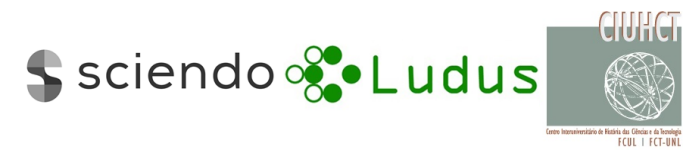

\title{
Articles
}

\section{OPTIMAL RECTANGLE PACKING FOR THE 70 SQUARE}

\author{
Brian Laverty, Thomas Murphy \\ West Virginia University, California State University Fullerton \\ blaverty91@gmail.com,tmurphy@fullerton.edu
}

\begin{abstract}
Gardner asked whether it was possible to tile/pack the squares $1 \times 1, \ldots, 24 \times 24$ in a $70 \times 70$ square. Arguments that it is impossible have been given by Bitner-Reingold and more recently by Korf-Mofitt-Pollack. Here we outline a simpler algorithm, which we hope could be used to give an alternative and more direct proof in the future. We also derive results of independent interest concerning such packings.
\end{abstract}

Keywords: packing problems, $70 \times 70$ square.

\section{Introduction}

Consider the equation

$$
1^{2}+2^{2}+\cdots+n^{2}=m^{2}
$$

where $n, m \in \mathbb{N}$. Since the work of Watson [5] in 1918, it is known that the only solutions are the trivial case, namely $1=1$, and

$$
1^{2}+\cdots+24^{2}=70^{2}
$$

A natural question arising from this fact is to see whether a configuration (or packing) of the squares $1 \times 1, \ldots, 24 \times 24$ exist which exactly tile a $70 \times 70$ square. The problem was popularized by Martin Gardner in articles 2, 3 in Scientific American, and several readers sent in their best efforts to fill as much of the square as possible. Twenty-seven readers managed to fit all the squares except the $7 \times 7$ square inside the $70 \times 70$ square; it has recently been proven 4 with the help of extensive computer computations that this is the 'best possible packing'. In particular, Korf et. al. showed it is impossible to construct a packing with the given squares which wastes less space. Their algorithm models the problem as a Constraint-Satisfaction Problem. This computation involved 375 million nodes, and took 16 minutes to compute (see Table 6 in the appendix of 4, as well as Section 4.5 for an overview of their algorithm). Each node is a placement of a set of squares in the enclosing 
rectangle, without overlap. An earlier article 1] states that applying their technique of backtrack programming shows that there is no such packing, but details are not provided due to the length of the arguments.

Our techniques and results are in a similar vein, and involve significant computations using MATLAB. Our goal is to give a mathematical proof, via a combination of combinatorial arguments and case-by-case analysis. Whilst we cannot yet fully achieve our goal, we outline an alternative approach using direct geometric arguments that we believe will yield a simpler proof in the future. Many of the results are applicable in wider contexts. To analyze the cases we could not directly rule out, we show how to rule out a packing with two fixed edges which were randomly chosen from the remaining possibilities. One could thus try to adapt our techniques and perform a case-by-case analysis to give a complete mathematical proof that no packing is possible. We hope to continue this work in the future.

\section{Results}

Assuming a packing exists of the $70 \times 70$ square, our goal will be to derive a contradiction. We will call this a potential packing, and for short denote it as $\mathcal{P}$. For the reader's convenience and to keep the diagrams a reasonable size, we will color in the $1 \times 1$ square in blue and the $2 \times 2$ squares in orange.

\section{Constructing the Matrix A}

Definition. An edge $\epsilon$ of $\mathcal{P}$ is a subset of $\mathcal{P}$ consisting of all squares which touch the same edge of the $70 \times 70$ square. The frame of $\mathcal{P}$ is the union of the four edges.

Let $a_{1}, \ldots, a_{n}$ be squares.

Definition. $\operatorname{ord}\left(a_{1}, \ldots, a_{n}\right)$ will denote an ordered set; this is a set of squares $\left\{a_{1}, \ldots, a_{n}\right\}$ so that $a_{k}$ and $a_{k+1}$ have adjacent sides for every $k=1, \ldots, n-1$.

Definition. Any permutation of an ordered set $\operatorname{ord}\left(a_{1}, \ldots, a_{n}\right)$ is denoted $\operatorname{adj}\left(a_{1}, \ldots, a_{n}\right)$.

The notation $\operatorname{adj}\left(a_{1}, \ldots, a_{n}\right)$ acts as a placeholder for multiple squares next to each other in an unordered fashion.

The following theorem restricts the number of possibilities significantly.

Theorem 1. (Restriction Theorem) Any edge of $\mathcal{P}$ must satisfy the following:

(i) The 1, 2, 3, 4, and 5 squares cannot be on any edge;

(ii) We cannot have the 6, 7, and 8-square on the same edge;

(iii) If the 6-square is on an edge, then we must have adj(6,7) on the same edge;

(iv) If $n$ is the smallest square on an edge, then the next smallest square on the edge must be less than $2 n-1$; 
(v) If $\operatorname{adj}(6,7)$ is on an edge, then the following construction must be on the same edge.

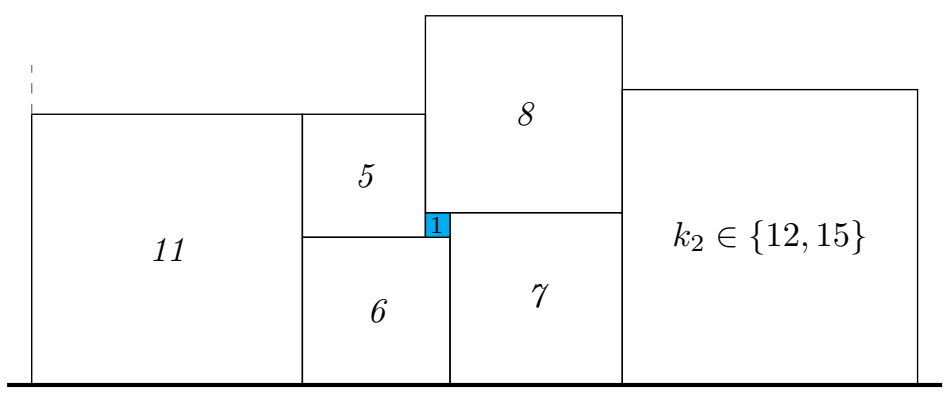

The next step, undertaken in Section 3 , is to construct a matrix $A$ where each row uses the numbers $6-24$ and sums to 70 ; these are all the possible sets of squares that can be on an edge of $\mathcal{P}$. To create this matrix, we first need to know how many squares (at most) are in a row of $A$; i.e., the number of columns in $A$. Since squares $1-5$ cannot lie on the edge, observing that

$$
6+7+8+9+10+11+12=64<70
$$

it follows that $A$ must have at most seven columns. To handle cases where, for example, one edge has seven squares and another has five, zeros must be included as placeholders to ensure MATLAB can display all possible edges as a matrix. The number of zeros is the maximum number of possible squares minus the minimum number of possible squares. Notice that $24+23+22+1=70$, so the minimum number of possible squares on an edge is four. After adding in the other four restrictions from Theorem 1 into our MATLAB code, this resulted in an entire column of zeros. Therefore, Theorem 1 ensures $A$ must have at most six columns; that is, there are at most six squares on an edge. Therefore there must be $6-4=2$ zeros included.

\section{Ruling Out Edges}

Our first attempt in MATLAB was to determine the matrix $A$ without any restrictions other than using 1-24 to sum to 70, i.e., not implementing Theorem 1. The closest we could reach to this was using 2-24 with seven columns which gave 9,285 rows. Applying all the restrictions from Theorem 1 to $A$, we obtain 391 rows.

We chose an element $v=\{9,14,23,24\} \in A$. This is the first entry of the matrix $A$. In Section 4 we will establish the following theorem:

Theorem 2. There is no frame with the edge

$$
v=\{9,14,23,24\}
$$

Many other potential edges occurring in $A$ can be ruled out via similar combinatorial arguments to the proof of Theorem 2 


\section{Constructing All Possible Frames}

In Section 5, we will produce an algorithm which produces all possible frames. We fix a row in $A$, called $v$, and find all rows in $A$ that have nothing in common with $v$ (excluding zeros). This new matrix is all possible sets of squares that are the opposite side of the $70 \times 70$ square; we denote this matrix by $A_{o p}$. Fixing a row in $A_{o p}$, denoted $v_{o p}$, a matrix $C$ is constructed from $A$ such that each row of $C$ has exactly one element in common with $v$ and $v_{o p}$ (not the same element). By finding two unique rows in $C$, then we have a possible frame. This is determined by fixing a row in $C$, denoted $c$, and determining all rows in $C$ with no common element with $c$. Finally, we construct the matrix $C_{o p}$ from $C$ by removing $c$ and any row that has a nonzero element in common with $c$ from $C$.

The matrix $A_{o p}$ is a subset of the matrix $A$. Thus we can only estimate the bound of $A_{o p}$ from our bound of $A$. After some numerical investigation, it appears that the bound for $A_{o p}$ appears to be approximately one third the bound of $A$, and thus the bound of $A_{o p}$ is approximately 3,000 before factoring in our restrictions. Applying Theorem 1 , the number of rows in $A_{o p}$ was reduced to 55 . Similarly, for the matrices $C$ and $C_{o p}$, the bounds will roughly be 660 and 224 respectively, before including restrictions. And after applying Theorem 1 the bounds of $C$ and $C_{o p}$ become 12 and 2, respectively.

In conclusion, we now have roughly $391 \times 55 \times 12 \times 3$ (roughly 770,000 ) possible frames to check. Given $\mathcal{P}$, its frame must be one of these possibilities.

\section{Ruling Out Frames}

To analyze our results, in Section 6 we rule out specific examples of frames which are obtained in our construction. This helps us to gain some intuition for how a proof would work in general.

Choosing the first edge $v$ where we cannot easily adapt the proof of Theorem 2 (which turns out to be the 80th possible entry of the matrix $A$ ), we randomly choose an element of $A_{o p}$ and show how no frame with these choices of $v$ and $v_{o p}$ is possible.

Theorem 3. There is no frame with edges

$$
\begin{aligned}
v & =\{7,8,14,18,23\}, \\
v_{o p} & =\{9,10,11,12,13,15\} .
\end{aligned}
$$

In the course of the proof, we reduce the number of possible frames with these two elements $(12 \times 3=36)$ to only nine cases, which are all easily handled by considering the possible ways of tiling around the smallest corner square. This leads us to believe our approach will work in general, which we leave as a project for the future.

\section{Near Misses}

We conclude with an interesting observation in Section 7, Running a direct calculation to find solutions of Equation 1, MATLAB ran out of memory when 
checking different $n$ values around $n=10^{6}$. The well-known formula

$$
1^{2}+2^{2}+\cdots+n^{2}=\frac{n(n+1)(2 n+1)}{6}
$$

cuts down on computation and allows us check more cases. Using MATLAB, there was a round-off error which reached the false conclusion that

$$
1^{2}+2^{2}+\cdots+2,542,690^{2}=2,340,882,545^{2} .
$$

We regard this as a "near-miss" solution, since the values on both sides of the equation differ by 1,140 ; which when divided by $m^{2}$ becomes minuscule. Defining the weighted error for given integers $n, m$ as

$$
E(n, m):=\left\{\frac{k}{m^{2}} \mid\left(1^{2}+\cdots+n^{2}\right)-m^{2}=k\right\}
$$

then our above example has a weighted error of a mere $2.08 \times 10^{-16}$. With this definition, we consider a pair $(n, m)$ to be a near-miss solution if $|E(n, m)|<10^{-15}$. Searching with MATLAB, we seem to find many more examples of near misses, which are recorded in the following table.

\begin{tabular}{|c|c|c|}
\hline$n$ & $m$ & $E(n, m)$ \\
\hline $2,542,690$ & $2,340,882,545$ & $2.08039 \times 10^{-16}$ \\
$3,179,535$ & $3,273,293,063$ & $1.11158 \times 10^{-16}$ \\
$3,344,320$ & $3,531,028,388$ & $-5.00474 \times 10^{-17}$ \\
$4,832,360$ & $6,133,076,209$ & $-7.49974 \times 10^{-17}$ \\
$5,988,346$ & $8,460,572,575$ & $-1.32213 \times 10^{-16}$ \\
$6,471,528$ & $9,504,946,458$ & $-1.08474 \times 10^{-16}$ \\
$7,050,120$ & $10,807,722,436$ & $-1.35917 \times 10^{-16}$ \\
$7,671,515$ & $12,267,642,825$ & $5.74769 \times 10^{-18}$ \\
\hline
\end{tabular}

It is interesting to ask if there are infinitely many pairs $(n, m)$ yielding near misses with less than a given error. If so, does the error approach zero as $n \rightarrow \infty$ ?

\section{Proof of Theorem 1}

The proof will be divided into a case-by-case analysis. To begin, we outline some definitions we will use in the proof.

Definition. Let $a, b \in \mathcal{P}$ be squares with $\operatorname{adj}(a, b)$. We say there is a flush of $a, b$ (or an $a, b$-flush) if there exists a square $c \neq a, b$ such that $\operatorname{adj}(a, c)$ and $\operatorname{adj}(b, c)$.

We write $|a|$ for the length of an $a \times a$ square. 
Definition. A set of squares $\Pi=\left\{a_{i}: i=1, \ldots, n\right\} \subseteq \mathcal{P}$ is called a path if $\Pi$ satisfies:

1. $\operatorname{ord}\left(a_{1}, \ldots, a_{n}\right)$,

2. $\sum_{i=1}^{n}\left|a_{i}\right|=70$, and

3. $a_{1} \in \epsilon$ and $a_{n} \in \epsilon_{o p}$ for some edges $\epsilon, \epsilon_{o p}$.

Definition. Let $a$ and $b$ be squares. We say $b$ is on top of $a$ if there exists a path $\Pi$ with $a, b \in \Pi$, so that $a=a_{i} \in \Pi$ and $b=a_{i+1} \in \Pi$ for some fixed $i$.

Note that the definition of "on top of" is deliberately flexible: performing a rotation allows us to view any given path as starting at the bottom of the square and proceeding vertically upwards.

Definition. Let $a$ and $b$ be squares with $b$ on top of $a$, where $|b|>|a|$ and $\operatorname{adj}(a, b)$ such that there exists an $a, b$-flush. Then $b$ will hang over $a$.

If the flush is the left then the hang over is on the right and vice-versa.

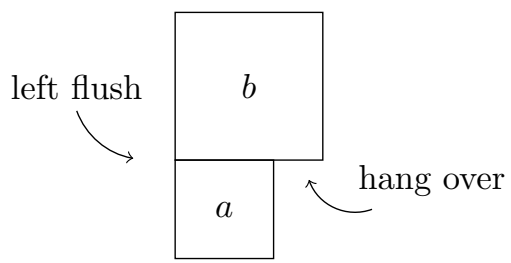

Figure 1: This illustrates an $a, b$-flush, and that $b$ is hanging over $a$.

\section{Proving Theorem 1|(i)}

Here we prove Theorem 1/(i), which states that squares 1-5 cannot be on the edge. We begin with a preliminary lemma to reduce the size of the proof. Owing to its length, the rest of the proof will be split up into a series of claims. Up to rotation we can assume an edge is on the bottom of the square. Without loss of generality the notation ord corresponds to squares being placed from left to right.

Lemma 1. Suppose the $1,2, \ldots, n-1$-squares are not on an edge. Then the $n$-square is not a corner square.

Proof. Suppose the squares $1,2, \ldots, n-1$ are not on an edge. Further, suppose for contradiction that the $n$-square is a corner square. Then there are adjacent squares $a_{1}, a_{2}$ on the corresponding edges. However, since squares $1, \ldots, n-1$ are not on an edge and the $n$-square was already used, then $\left|a_{1}\right|,\left|a_{2}\right|>|n|$ which guarantees an overlap and thus a contradiction.

Throughout there will be diagrams of square packings. To help see the larger packings, we color the 1-square with blue and color the 2-square with orange throughout the paper. We also use the symbol $\times$ in the diagrams to illustrate where a contradiction occurs. 
Claim. The 1-square cannot be on the edge.

Proof. Suppose the 1-square is on the edge. Let squares $a_{1}, a_{2}$ be squares on the same edge where (without loss of generality) $1<\left|a_{2}\right|<\left|a_{1}\right|$ are on the right and left of the 1-square, respectively. Then, any remaining square placed on top of $a_{1}$ will yield wasted space above the 1 -square. The 1 -square cannot be a corner square by a similar argument as Lemma $1 . \rightarrow \leftarrow$

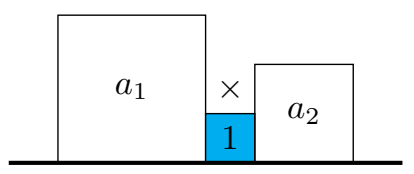

Claim. The 2-square cannot be on the edge.

Proof. Suppose the 2-square is on the edge. By Lemma 1, the 2-square is not a corner square. Let $b_{1}, b_{2}$ be squares on the edge such that (without loss of generality) $2<\left|b_{2}\right|<\left|b_{1}\right|$. Place $b_{1}$ and $b_{2}$ on the left and right of the 2-square, respectively. Then the set of squares on the 2-square must sum to two. However, the 1 -square is the only square less than the 2 -square remaining and this yields wasted space. $\rightarrow \leftarrow$

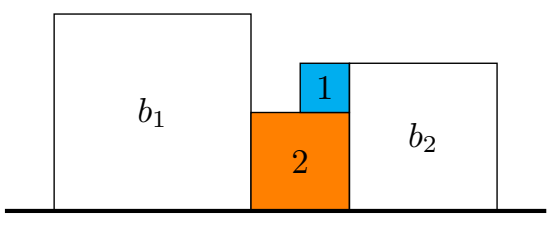

Claim. The 3-square cannot be on the edge.

Proof. Suppose the 3-square is on the edge. By Lemma 1, the 3-square is not a corner square. Let $c_{1}, c_{2}$ be squares on the edge where $3<\left|c_{2}\right|<\left|c_{1}\right|$. Place $c_{1}$ and $c_{2}$ on the left and right (without loss of generality) of the 3 -square, respectively. Then the only squares that can be placed on top of the 3-square is $\operatorname{adj}(1,2)$. Then for any $\left|c_{2}\right|>4$, we get wasted space over the 1-square for either permutation of $\operatorname{adj}(1,2)$. Thus, $c_{2}$ must be the 4 -square, and the 1 -square must be on the right. Similarly, for any $\left|c_{1}\right|>5$, there will be wasted space over the 2-square.

Now consider if the 4-square is a corner square. Then any square placed on to the 1,4-flush must be $\geqslant 6$ (since squares $1-5$ have been used). However, this guarantees overlap with the 2 -square. This implies that there must be a square $c_{3}$ where $\left|c_{3}\right| \geqslant 6$, on the right of the 4-square on the edge. Thus any set of squares placed on top of the 1,4-flush must sum to five, but there are no remaining sets that sum to five. $\rightarrow \leftarrow$ 


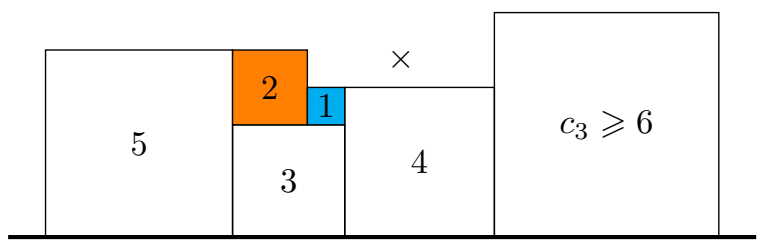

Claim. The 4-square cannot be on the edge.

Proof. Suppose the 4-square is on the edge. By Lemma 1 the 4-square is not a corner square. Let $d_{1}, d_{2}$ be squares on the edge where $4<\left|d_{2}\right|<\left|d_{1}\right|$. Place $d_{1}$ and $d_{2}$ on the left and right (without loss of generality) of the 4-square, respectively. So the set of squares on the 4 -square must sum to four. The only set of squares that satisfies this sum is $\operatorname{adj}(1,3)$, so place this on top of the 4-square. Notice that for any $\left|d_{2}\right|>5$, there will be wasted space over the 2 -square. So $d_{2}$ must be the 5 -square, and to avoid wasted space we must have $\operatorname{adj}(1,5)$.

Since squares $3-5$ have been used then any edge square placed adjacent to the 5 -square will be larger than five, and so the set of squares on the 1,5-flush must sum to six (this also holds if the 5 -square is a corner square). The 6 -square is the only square that can be placed on the 1,5-flush; make this placement. Note that $\left|d_{1}\right| \geqslant 7$. Consider when $\left|d_{1}\right|>7$, then the 2 -square is the only remaining square that can be placed on the 3-square. However, this guarantees wasted space adjacent to the 2 -square. Thus $d_{1}$ must be the 7 -square.

Consider if the 5 -square is a corner square. Then any square placed on top of the 6 -square and on this new edge must be $\geqslant 8$, and will therefore hang over the 6 -square. However, the 2 -square is the only remaining square that can be placed on the 3 -square, and this guarantees wasted space adjacent to the 2 -square. Thus, there must be another square $d_{3}$ on the right of the 5 -square. This further implies that any set of squares placed on the 6-square must hang over the 6 -square on the right.

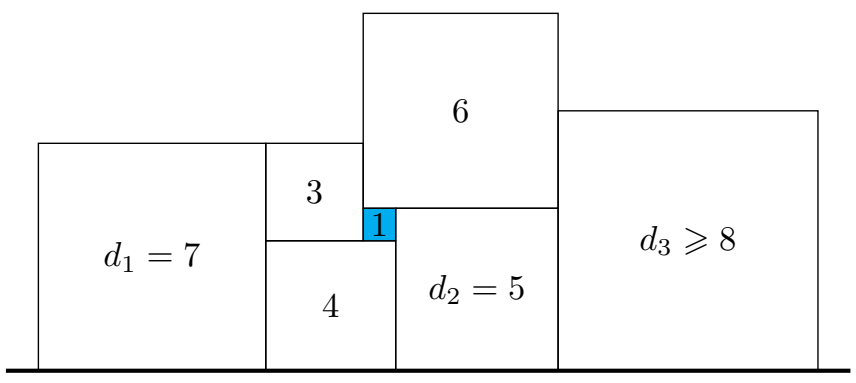

Since the set of squares on the 6-square will hang over on the right, then the set of squares adjacent to the 5,6-flush must sum to 11 . The only remaining sets are $\operatorname{adj}(2,9)$ and the 11 -square. Consider when $d_{3}$ is the 9 -square with the 2 -square placed on top. Then we must have the 8-square placed on the 2,6-flush otherwise there will be wasted spaced to the right of the 2-square. Further, any square placed on the right of the 9-square will be larger than nine, so the 
(remaining) set of squares on the 9-square must sum to nine-this also holds if the 9-square is a corner. However, there are no remaining squares less than nine. So $d_{3}$ cannot be the 9 -square and therefore must be the 11 -square.

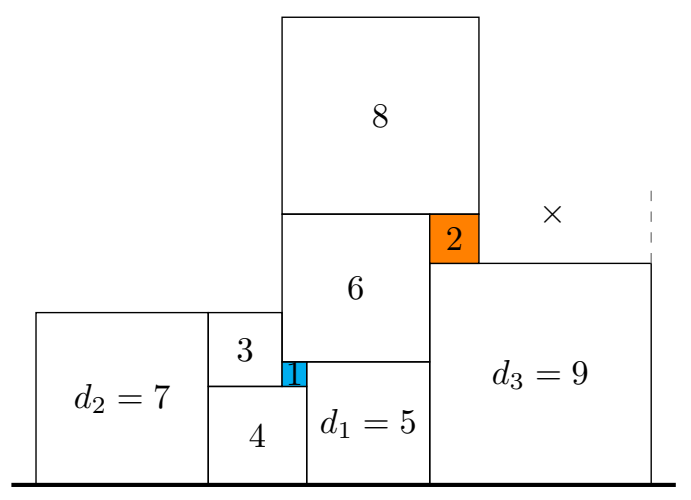

Next place another square $d_{4}$ on the left of the 7 -square. Further, since squares $3-7$ have been used then $\left|d_{4}\right| \geqslant 8$. It is possible that the 7 -square is a corner square; we will modify our arguments if necessary on a case-by-case basis. Therefore the set of squares placed on top of the 3,7-flush must sum to ten. The only remaining sets that satisfy this are $\operatorname{adj}(2,8)$ and the 10 -square.

Consider when $\operatorname{adj}(2,8)$ are placed on the 3,7-flush. Then the 2-square must be on the left, otherwise there would be wasted space over the 2 -square. Note that $\left|d_{4}\right| \geqslant 9$, but if $\left|d_{4}\right|>9$, then there will be wasted space over the 2-square; so $d_{4}$ must be the 9-square. Again, since squares 1-9 have been used, any square placed on the left of the 9 -square on the edge will be greater than nine. So the set of squares on the 2,9-flush must sum to eleven-this also holds if the 9 -square is a corner. But there are no remaining sets that sum to eleven. Thus, we cannot have $\operatorname{adj}(2,8)$ on the 3,7 -flush.

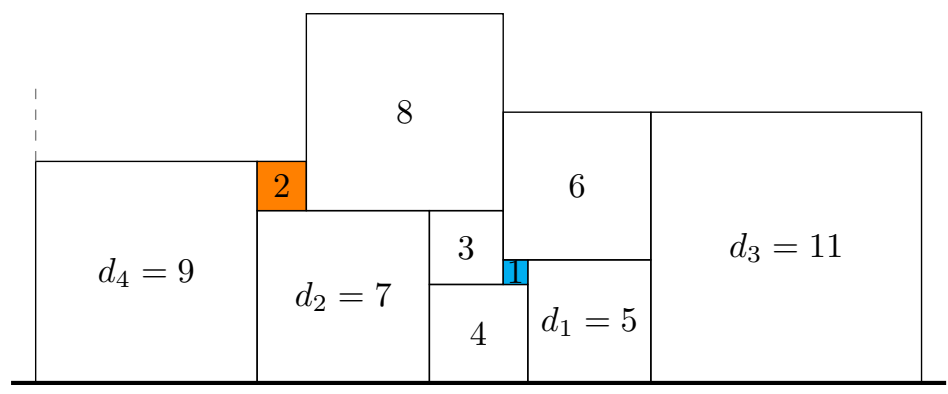

Therefore, the 10-square must be placed on the 3,7-flush. Note the following argument will also hold if the 7 -square is a corner. Place another square $d_{5}$ on top of the 6,11 -flush. If $d_{5}$ is the 2 -square, then this will yield wasted space adjacent to the 2 -square; so $\left|d_{5}\right| \geqslant 8$. Then we have two cases to consider: the set of squares on the 10-square either sums to ten or will hang over 10-square. 


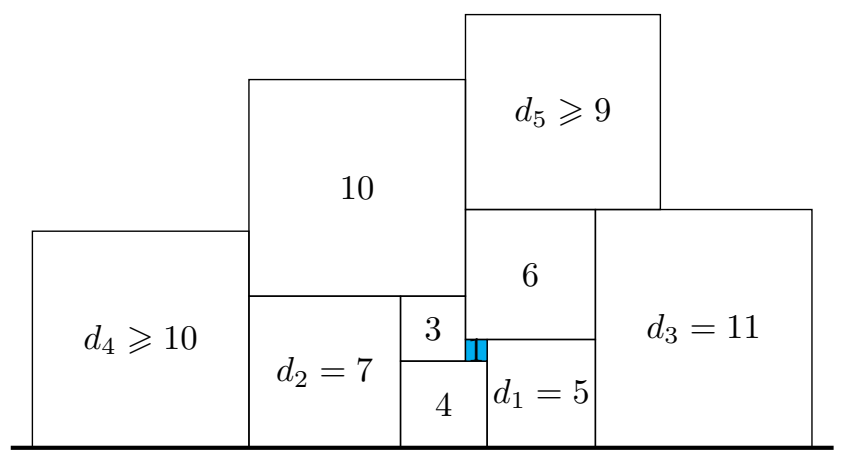

Note that the 8-square can no longer be on the edge since it is the smallest remaining square that can be placed on the edge but there are no remaining sets of squares that sum to eight. Similarly, the 9-square also cannot be on the edge since the set of squares on the 9 -square would need to sum to nine, and there are no such remaining sets.

1. Suppose the set of squares on the 10 -square sums to ten; $\operatorname{adj}(2,8)$ is the only remaining set that sums to ten. The 2 -square must be on the left otherwise there will be wasted space over the 2-square (for any square placed on the 6-square). Note that the set of squares adjacent to the $2,7,10$-flush must sum to 19 , otherwise there will be wasted space over the 2 -square. Further, the 19-square is the only remaining square that sums to 19 ; so $d_{4}$ must be the 19 -square.

Recall the 9-square cannot be on the edge. Thus if a square is on the right of the 11-square must be greater than 11 . Therefore the set of squares on the 6,11 -flush must sum to 17 (this holds if the 11 -square is a corner square); the 17-square is the only remaining square that satisfies this sum. However, any square placed on the 2-square will yield wasted space over the 8 -square. Thus, we cannot have $\operatorname{adj}(2,8)$ on the 10 -square, and therefore the set of squares on top of the 10-square must hang over the 10-square.

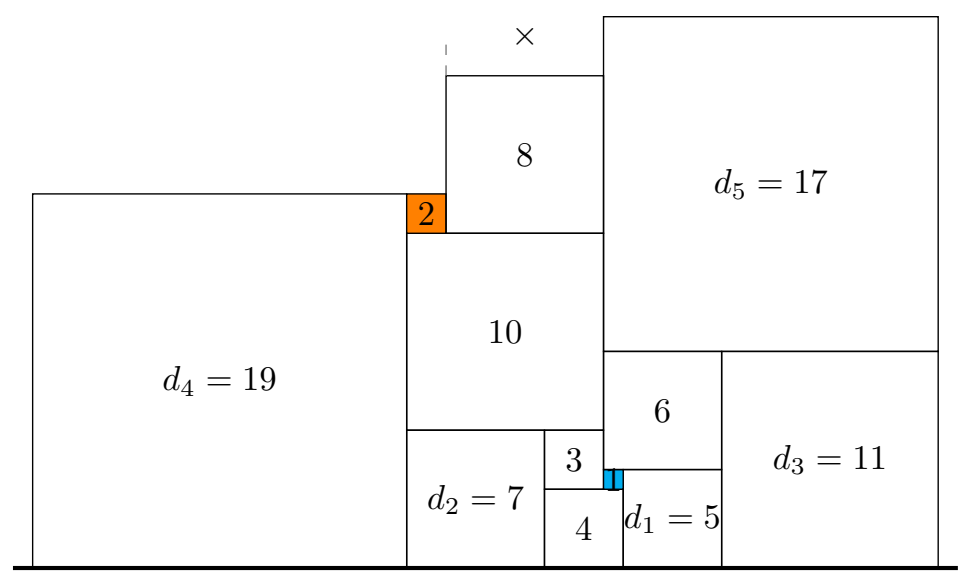


2. Suppose the set of squares on the 10-square hangs over the 10-square. Note that the 7-square cannot be a corner square or this would contradict our assumption. Further, the set of squares adjacent to the 7,10-flush must sum to 17 . The only sets that satisfy this $\operatorname{are} \operatorname{adj}(2,15), \operatorname{adj}(8,9)$, and the 17-square. However, we determined before that the 8 and 9 -squares cannot be edge squares. Further, we need a set of squares that sums to 17 to be placed on the 6,11-flush.

(a) Suppose $d_{4}$ is the 15 -square and place the 2-square on top of it. Note that there are no remaining sets of squares that sums to 15 , so the set of squares on top of the 15-square must sum to 15 (this also holds if the 15-square is a corner square). That is, the remaining set of squares on the 15 -square must sum to 13 ; the 13 -square is the only square that satisfies this sum. Note any square placed on the 6-square will have a height above the 10-square. So the set of squares on the 2,10 -flush must sum to 12 , and the 12 -square is the only square that satisfies this. Further, any square placed on the 12-square will hang over the 12-square and yield wasted space on the respective side. Thus, $d_{4}$ cannot be the 15 -square.

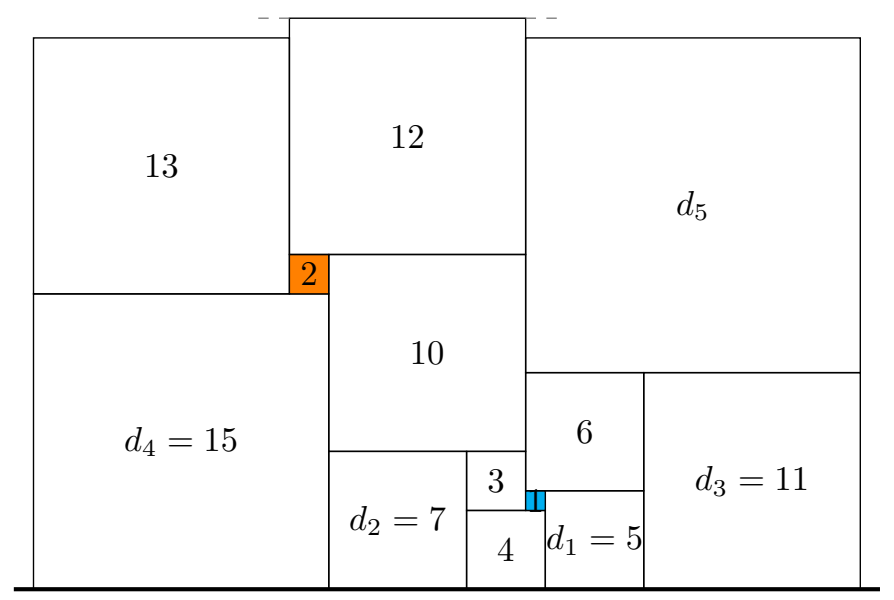

(b) Suppose $d_{4}$ is the 17 -square and adj $(8,9)$ is placed on the 6,11 -flush. Further, suppose the 9 -square is on the left. Then any square placed on the 9-square will hang over on either side, yielding wasted space. Similarly, if the 8-square is on the left, any square placed on top will hang over on the left. This guarantees wasted space over the 10 -square. So adj $(8,9)$ cannot be on top of the 6,11-flush. Then $\operatorname{adj}(2,15)$ must be on the 6,11-flush; the 2 -square must be on the right otherwise there will be wasted space over the 2 -square. Then we must have the 13 -square on the right of $d_{3}$ or there will be wasted space over the 2-square. Since there is remaining set that sums to 13 , then we must have the set of squares on the 2,13-flush must sum to 15 . But there are also no remaining sets of squares that sum to 15. Therefore, there will be wasted space over the 2,13-flush. Thus, $d_{4}$ cannot be the 17 -square. 


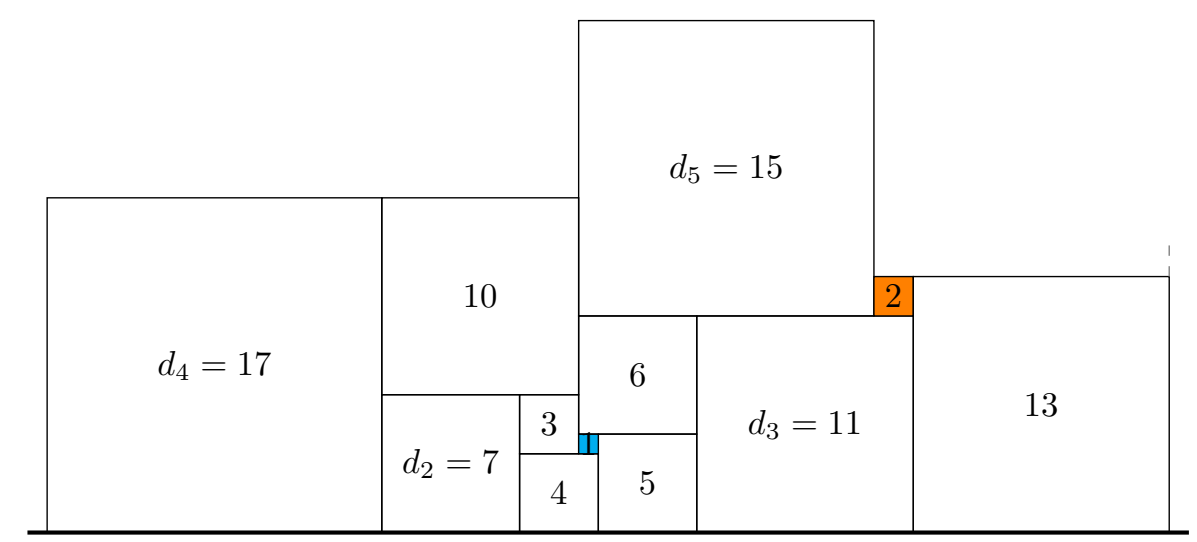

In summary, we cannot have the set of square on the 10-square sum to ten nor can they hang over the 10-square. We have a contradiction, and thus the 4-square cannot be on the edge.

Claim. The 5-square cannot be on the edge.

Proof. Suppose the 5-square is on the edge. By Lemma 1 the 5 -square is not a corner square. Let $e_{1}, e_{2}$ be squares on the edge where $5<\left|e_{2}\right|<\left|e_{1}\right|$. Place $e_{1}$ and $e_{2}$ on the left and right (without loss of generality) of the 5 -square, respectively. So the set of squares on the 5 -square must sum to five. The only sets that satisfies this sum $\operatorname{are} \operatorname{adj}(2,3)$ and $\operatorname{adj}(1,4)$.

1. Suppose $\operatorname{adj}(2,3)$ is placed on top of the 5 -square with the 2 -square on the right. If $\left|e_{2}\right|>7$ then any set of squares placed on the 2-square will yield wasted space. If $e_{2}$ is the 6 -square, then any set of square placed on the 2 -square will hang over the 2 -square. So place the 1 -square on the 6 -square, making a 1,2-flush. However, any square placed on the 2-square will hang over the 1-square, yielding wasted space. Thus, $e_{2}$ must be the 7-square.

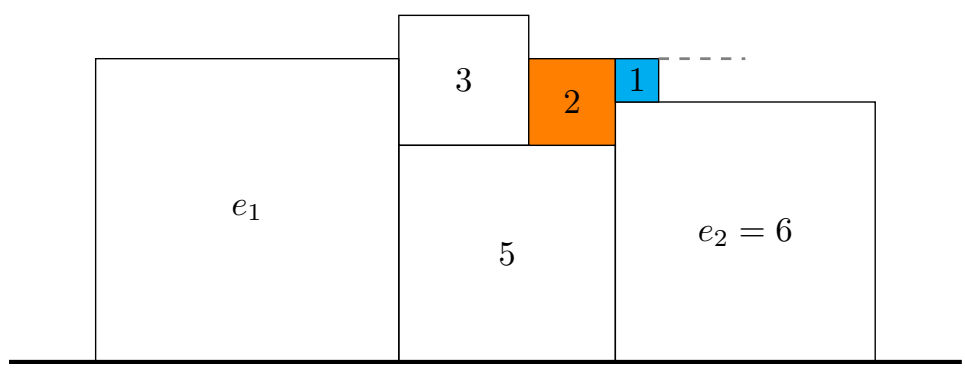

Note $e_{1}$ cannot be the 6 -square since $5<\left|e_{2}\right|<\left|e_{1}\right|$. Consider when $e_{1}$ is the 9 -square, then any set of squares on the 3 -square will hang over the 3 -square. The only configuration that will not (immediately) yield wasted space is to place the 1 -square on the 2 -square, and place the 4 -square on the 1,3-flush. However, we must have the set of squares on the 2,7-flush sum to nine, otherwise there will be wasted space next to the 7 -square. So 
we must have $\operatorname{adj}(1,8)$ on the 2,7 -flush. However, then any square placed on the 4-square will hang over the 4-square and leave wasted space under the overhang. Thus $e_{1}$ cannot be the 9 -square.

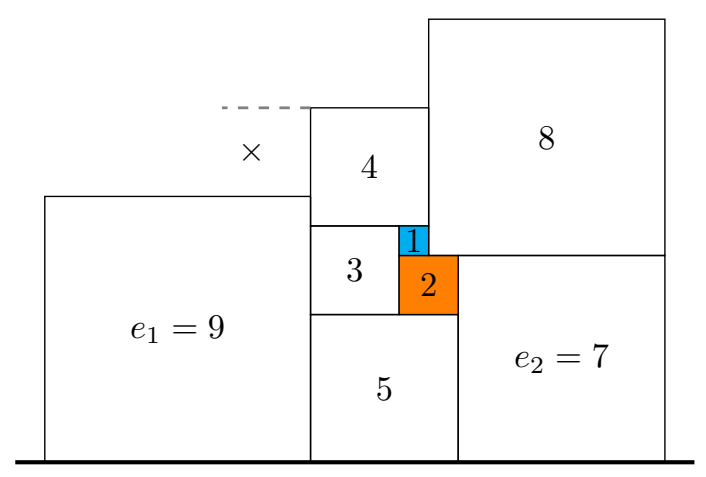

Now, we have the remaining cases: $\left|e_{1}\right|>9$ and $\left|e_{1}\right|=8$. Suppose $\left|e_{1}\right|>9$ then we see from the case $e_{1}$ was the 9 -square, that we must have the 1-square on the 2-square making a 1,3-flush and the 4 -square on top of the flush. Now any square placed on the 4-square will hang over the 4 -square, so $e_{1}$ must be the 12 -square since no other sets sum to twelve. Again from the previous case, we know that the set of squares on the 2,7-flush must sum to nine and so the 8-square must be placed on the 2,7-flush.

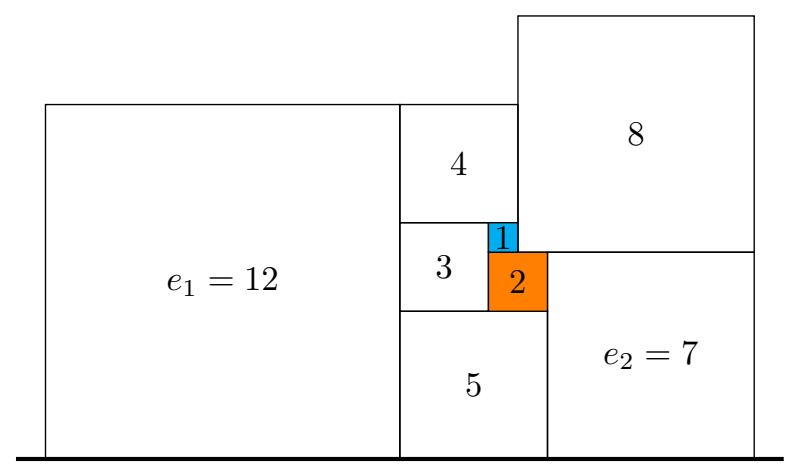

Since there are no remaining sets of squares that sum to eight, then the set of squares on top of 8-square will hang over the 8-square. But any square placed on the 4 -square will have a height above the 8 -square, so the overhang must be on the right. This implies that $e_{2}$ cannot be a corner square. Therefore, we need the squares adjacent to the 7,8 -flush to sum to 15 . The only squares that satisfy this are $\operatorname{adj}(6,9)$ and the 15 -square.

Consider when the 9-square is on the right of the 7 -square, with the 6 -square placed on top of the 9-square. Then any square on the right of the 9-square will be larger than the 9-square. But there are no remaining squares that can be placed adjacent to the 6 -square. So we cannot have $\operatorname{adj}(6,9)$ on the 7,8 -flush. 


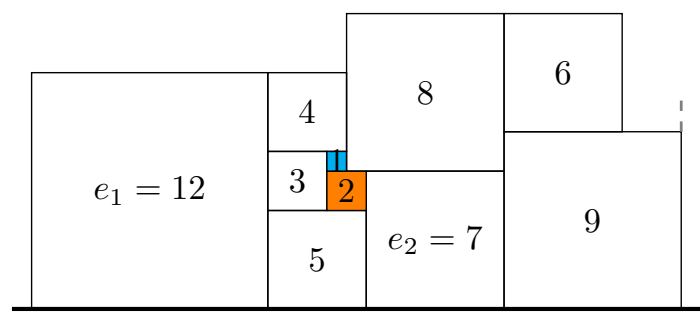

We must have the 15 -square on the right of the 7 -square. Consider the total length of these squares on the edge, $12+5+7+15=39$. The remaining length on the edge is $70-39=31$. From the remaining squares, the possible combinations are $\operatorname{adj}(6,11,14)$, adj $(9,22), \operatorname{adj}(10,21)$, $\operatorname{adj}(11,20), \operatorname{adj}(13,18)$, and $\operatorname{adj}(14,17)$. Note that $\operatorname{adj}(6,11,14)$ is ruled out since no remaining set of squares sum to 16 . So all of these sets can be represented $\operatorname{as} \operatorname{adj}(a, b)$ where $9 \leqslant|a|<|b|$.

Consider the smaller square $a$ in each of these sets. For any placement on the edge, the set of squares on $a$ must sum to $|a|$. Note that the 6 and 9 -squares are the two smallest remaining squares, so $|a| \geqslant 15=6+9$. However, $a$ is at most the 14 -square. Therefore this guarantees wasted space over $a$ in any of these sets. Thus, we cannot have $\operatorname{adj}(7,15)$.

For example, if $\operatorname{adj}(14,17)$ were placed on the edge, then there must be another set of squares to be placed on the 14-square. But the next smallest squares are the 6 and 9 -square, so this guarantees wasted space over the 14-square (the image below is simply a visual aide; further, the argument holds for any placement of $a$ and $b$ on the remaining edge).

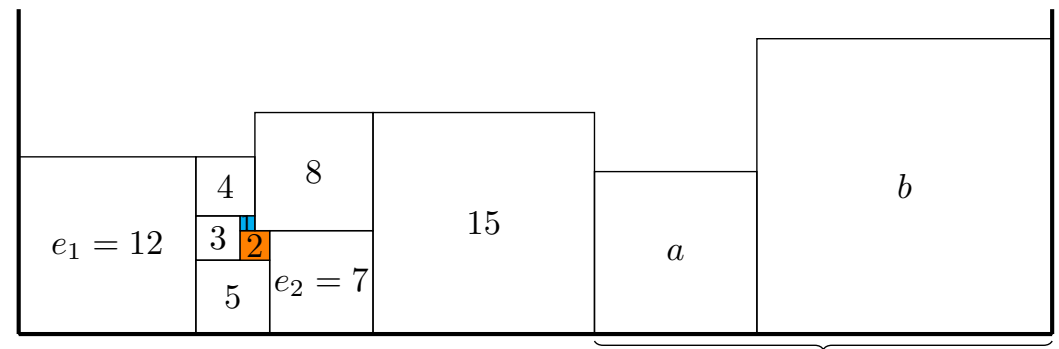

31

Thus, $e_{1}$ cannot be the 12-square. In summary, we have ruled out $\left|e_{1}\right|=6$ and $\left|e_{1}\right| \geqslant 9$. So $e_{1}$ must be the 8-square. Again, we must have the set of squares on the 2,7-flush sum to nine. But the 9 -square is the only square that satisfies this sum. So place the 9 -square on the 2,7-flush. We place another square on the left of the 8-square denoted $e_{3}$. Note that $\left|e_{3}\right| \geqslant 10$, since any set of squares on the 6 -square would yield wasted space and squares 7-9 have been used. Then the set of squares on the 3,8-flush must sum to 11 . The only sets that satisfy this are $\operatorname{adj}(1,10), \operatorname{adj}(1,4,6)$, and the 11-square. 


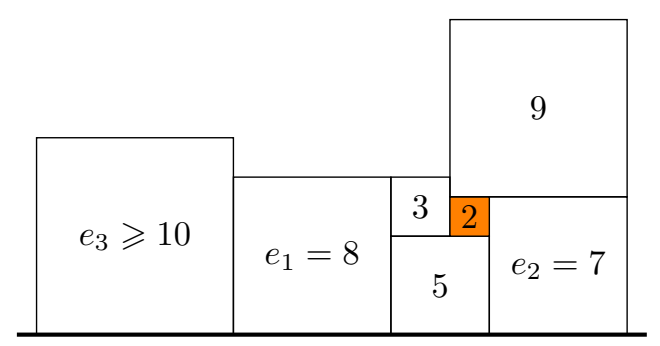

Consider the cases when $\operatorname{adj}(1,10)$ and $\operatorname{adj}(1,4,6)$ are placed on the 3,8 -flush. Then any permutation will guarantee wasted space over the 1-square. Therefore the 11-square must be on the 3,8-flush. Note that if the 8-square is a corner square then these first two cases would have the same wasted space. Note that there are no remaining sets of squares that sum to eight or nine. Then any set of squares placed on top of the 9-square will hang over the 9 -square on the right. This implies the 7 -square is not a corner square, and the set of squares adjacent to the 7,9-flush must sum to 16 . Place a square, $e_{4}$, on the right of $e_{2}$. The only remaining sets that sum to 16 are $\operatorname{adj}(1,15), \operatorname{adj}(4,12), \operatorname{adj}(6,10)$, and the 16 -square.

We therefore have the following cases: the set of squares on the 11-square either sums to 11 or hangs over the 11-square. Now, consider the particular case when the 4 -square is placed on the 9 -square and the 1-square is placed on the 11-square; this creates a 1,4-flush. But any squares on the right of the 4-square and left of the 1-square will yield wasted space above the 1,4-flush. Further, if the 1-square is placed on the 9-square there will be wasted space over the 1-square. Therefore, any square placed on the 9 -square will have a height above the 11-square.

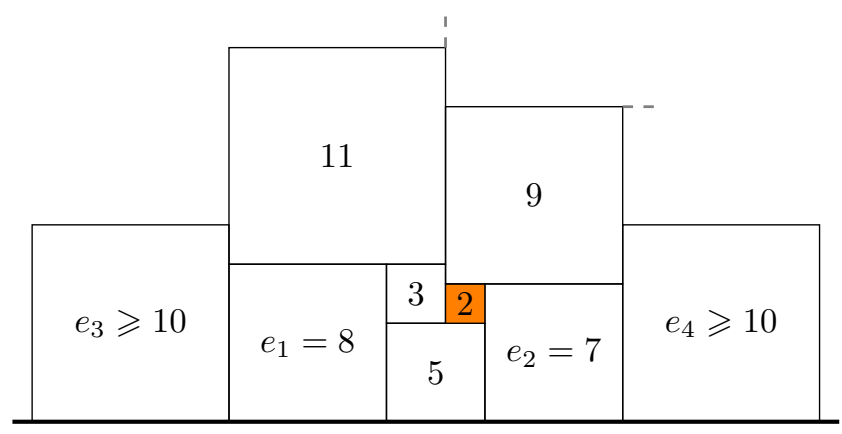

(a) Suppose the set of squares on the 11-square sums to 11 . Then the only sets that satisfy this are $\operatorname{adj}(1,4,6)$ and $\operatorname{adj}(1,10)$. Consider when $\operatorname{adj}(1,4,6)$ is placed on the 11 -square, then we have either ord $(1,4,6)$, ord $(1,6,4)$ or ord $(4,1,6)$. However, ord $(4,1,6)$ guarantees wasted space over the 1-square, and ord $(1,4,6)$ guarantees wasted space over the 1 -square for any square placed on top of the 4-square. Further, for $\operatorname{ord}(1,6,4)$, any square placed on the 6 -square will hang over the left or right of the 6 -square which will guarantee wasted space over the 1 or 4-square accordingly. 
So place $\operatorname{adj}(1,10)$ on the 11-square, with the 1-square on the left since we've shown that the 1-square cannot be on the right. Then the set of squares on the 8,11,1-flush must sum to 20 , to avoid wasted space over the 1 -square. The only sets that satisfy this are $\operatorname{adj}(4,16)$, $\operatorname{adj}(6,14)$, and the 20 -square. But we cannot have $\operatorname{adj}(4,16)$ since this removes the remaining sets on the right of the 7 -square.

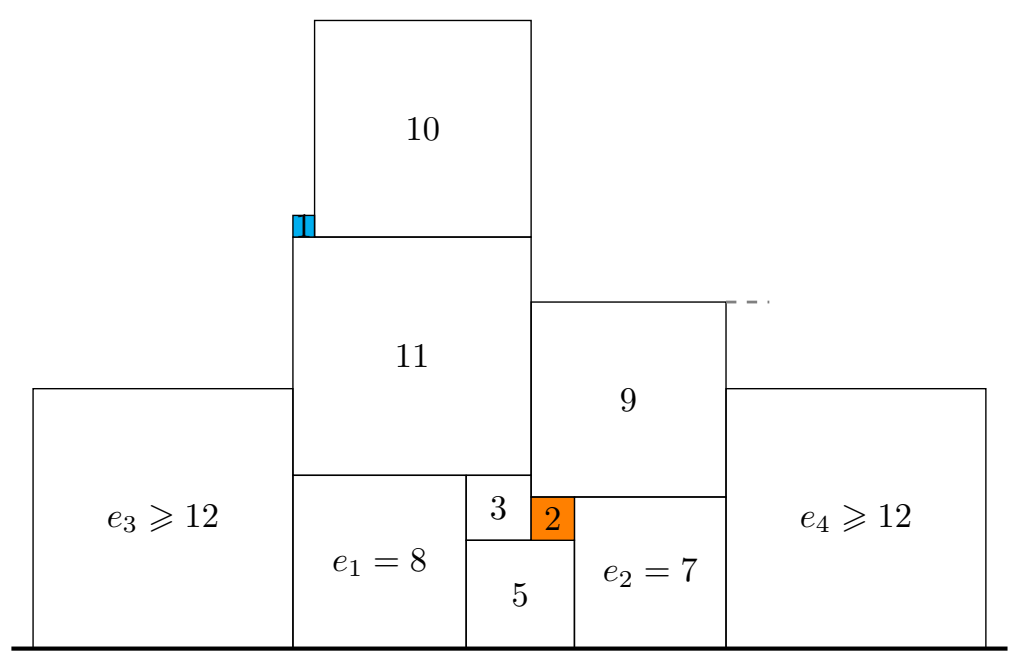

Suppose $e_{3}$ is the 14 -square and place the 6 -square on top of it. Then any square placed adjacent to the 6 -square will hang over the 14-square. And there are no remaining sets that sum to 14 . Thus, $e_{3}$ cannot be the 14 -square, and so $e_{3}$ must be the 20 -square.

Consider if the $e_{4}$ is the 16-square. Then the total length of squares on the edge is $20+8+5+7+16=56$; so there remaining squares must sum to 14 . However, there are no remaining sets that sum to 14 . Thus, $e_{3}$ cannot be the 16 -square (the image shows the 14-square on the right of the placed squares without loss of generality; the argument holds if the 14-square is on the left as well).

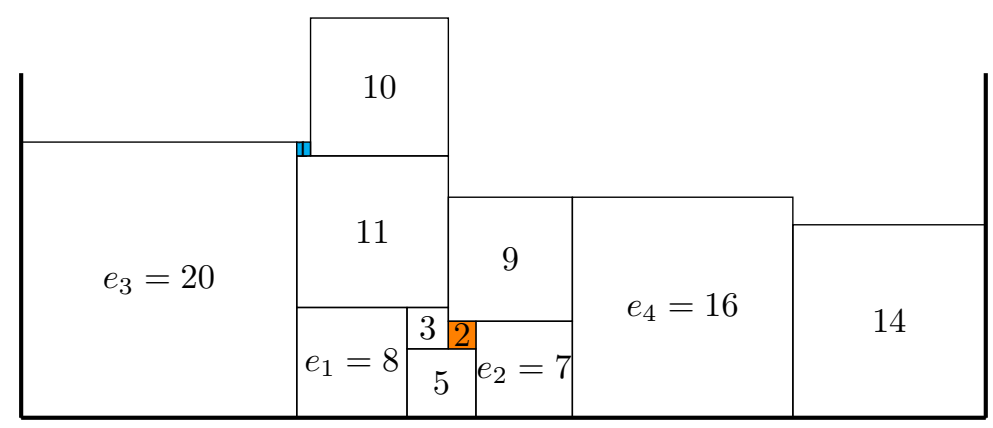


Let $e_{4}$ be the 12 -square and place the 4 -square on top of it. Then any squares placed on the remaining space on the 12-square will hang over the 12-square. However any square placed on the right of the 12-square will be greater than 12 . Thus, $e_{4}$ cannot be the 12 -square.

Thus we cannot have the set of squares on the 11-square sum to 11 .

(b) Suppose the set of squares on the 11-square hangs over the 11-square. Then the set of squares adjacent to the 8,11-flush must sum to 19 . The only sets that satisfy this $\operatorname{are} \operatorname{adj}(1,18), \operatorname{adj}(4,15), \operatorname{adj}(6,13)$, $\operatorname{adj}(1,4,14)$, and the 19-square.

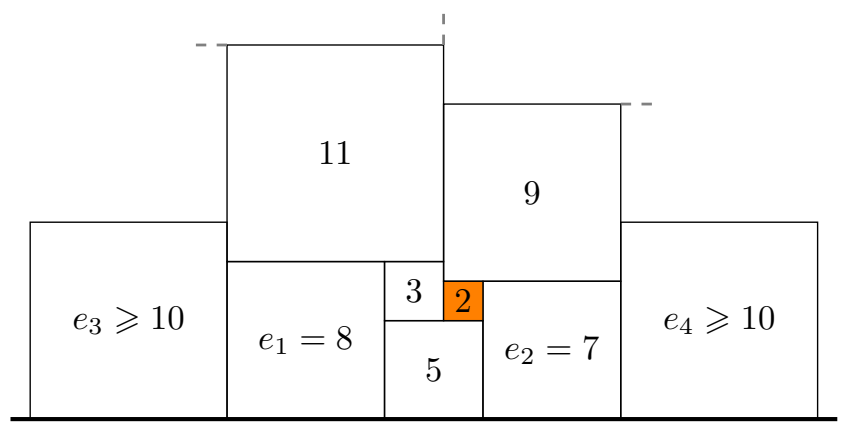

i. Suppose $e_{3}$ is the 18-square and place the 1-square on top of it. Now, the only sets of squares that adjacent to the 7,9-flush that sum to 16 are adj $(6,10)$ and the 16 -square. We again consider the total length of the squares on the edge used: $18+8+5+7=38$. So we have $70-38=32$ remaining length on the edge. If $e_{4}$ is the 16 -square then there is a length of 16 remaining, but adj $(6,10)$ is the only set of squares that would fit this space, but the 6 -square will yield wasted space above it.

Further, if $e_{4}$ is the 10-square then there is a length of $70-(18+8+5+7+10)=22$ remaining on the edge. We must have the set of squares on the 10-square sum to ten to avoid wasted space over the 10 -square. Place ord $(4,6)$ on the 10 -square with the 6 -square on the left (otherwise there will be wasted space over the 4 -square). If the 10 -square is a corner square then there will be wasted space over the 4 -square. This implies that there must be a square to the right of the 10-square; the 14-square is the only square that can be placed to the right of the 10-square without wasted space. This however leaves a remaining length of $22-14=8$. Therefore $e_{3}$ cannot be the 18-square (the image shows the remaining length on the right without loss of generality). 


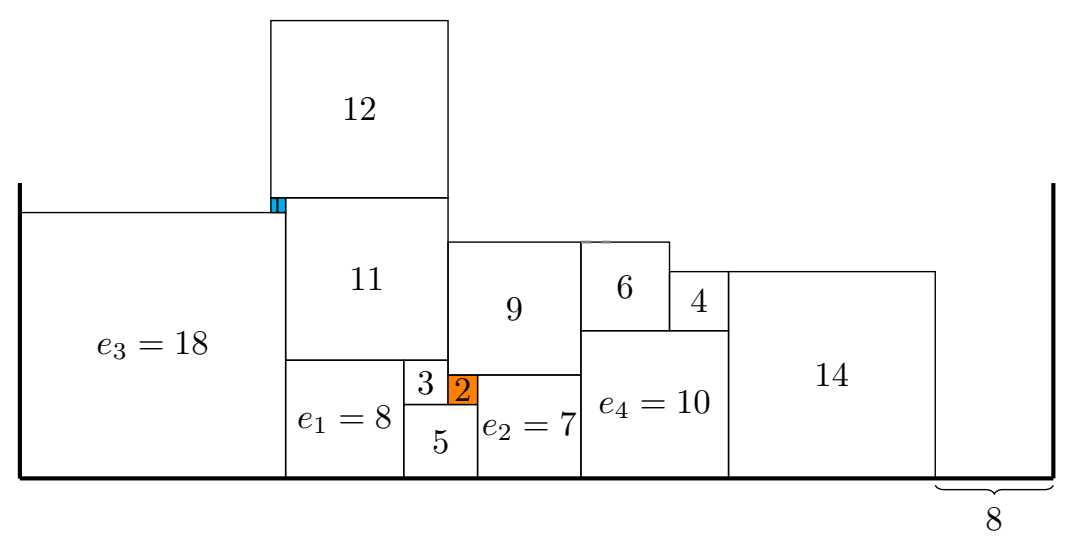

ii. Suppose $e_{3}$ is the 15 -square and place the 4-square on top of it. Then any square placed on the left of the 4-square will be greater than the 4-square (the 1-square would yield wasted space). Also any set of squares placed on the 9-square will have a height above the 11-square. We need the set of squares on the 4,11-flush to sum to 15 . The only set that satisfies this is adj(1,14). This however will yield wasted space over the 1-square for either permutation. Thus, $e_{3}$ cannot be the 15 -square.

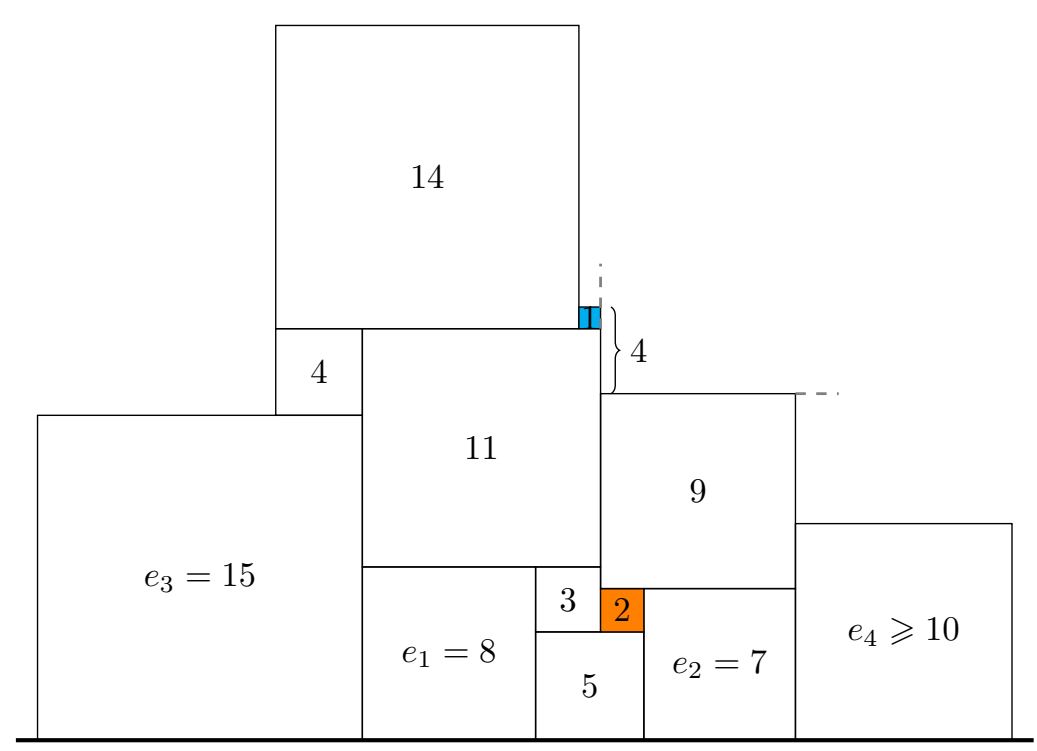

iii. Suppose $e_{3}$ is the 13 -square and place the 6-square on top of it. Then suppose the 12-square is placed on the left of the 13 -square. Then there are no sets that sum to 12 that can be placed on top of the 12-square. So the square on the right of the 13-square must be greater than 13. That is, the set on top of the 13 -square must sum to 13 . Since the 6 -square is already placed on the 13-square then we need a set that sums to 7 , but there is no such set of squares remaining. Thus, $e_{3}$ cannot be the 13 -square. 
iv. Suppose $e_{3}$ is the 14 -square, place the 4 -square on top of the 14-square, and place the 1-square on top of the 4-square. Then any square placed next to the 1-square will hang over the 4-square leaving wasted space. So, $e_{3}$ cannot be the 14-square.

v. Suppose $e_{3}$ is the 19-square. Consider the length of squares on the edge so far, we have $19+8+5+7+\left|e_{4}\right|=39+\left|e_{4}\right|$. Observe the set of squares placed on the 9 -square will hang over the 9-square. Therefore the set of squares on the 7,9 -flush must sum to 16 . That is, we must have one of $\operatorname{adj}(6,10)$, $\operatorname{adj}(4,12), \operatorname{adj}(1,15)$, or the 16 -square on the 7,9 -flush. Note the 6 -square cannot be on the edge since no remaining sets sum to six. Suppose $e_{4}$ is the 10-square then, similarly to the first case, we must have $\operatorname{adj}(4,6)$ on the 10 -square and the 14-square must be to the right of the 10-square. This leaves a length of 7 remaining on the edge, which guarantees wasted space.

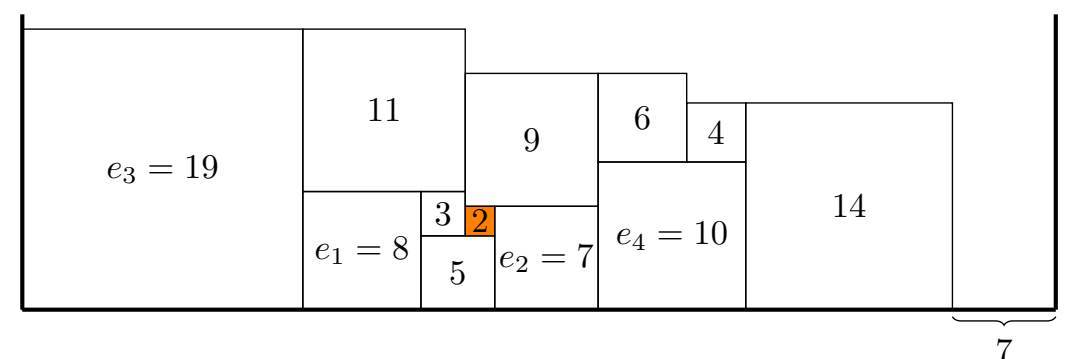

Suppose $e_{4}$ is the 12 -square. Then the remaining total gap is 19. The only sets of squares that can fill this is $\operatorname{adj}(1,18)$ and $\operatorname{adj}(6,13)$. However neither of these sets can be placed on an edge.

Suppose $e_{4}$ is the 15 -square. There is then a total gap of 16 remaining on the edge. Since the 6 -square cannot be on the edge then the 16-square must fill this gap. We consider when the 16-square is to the right of the 15 -square. Then the set of squares on top of the 15-square must sum to 15 . The only possible set is $\operatorname{adj}(1,14)$. We must have ord $(1,14)$ since no remaining sets sums to nine. However, this yields wasted space over the 16-square. An easier, analogous argument shows we cannot have the 16-square on the left of the 19-square.

Now supposing $e_{4}$ is the 16-square, then the remaining length on the edge is $70-55=15$. The 15 -square is the only square that can be placed in the remaining edge. The only sets remaining that sum to 15 are $\operatorname{adj}(1,14)$ and $\operatorname{adj}(1,4,10)$.

Consider when $\operatorname{adj}(1,4,10)$ is placed on the 15 -square. Then the 1-square must be on the right to avoid wasted space. Further, the 15-square must be a corner square since we have used the entire edge. Therefore the 10-square must be on the corresponding edge. However, any square placed to the right of the 10-square (on the new edge, call this $\epsilon_{2}$ ) must be larger 
than the 10 -square. So we must have adj $(4,6)$ on the 10 -square. However, this guarantees wasted space over the 1-square. Therefore $\operatorname{adj}(1,14)$ must be on the 15 -square.

Suppose the 13-square is placed on the 1-square. Then there are no remaining sets that sum to 13 . So there will be wasted space above the 9,16 -flush. So, the 13 -square cannot be placed on the 1-square. Now, the remaining sets that sum to 26 are $\operatorname{adj}(4,22), \operatorname{adj}(6,20)$, and $\operatorname{adj}(4,10,12)$. If $\operatorname{adj}(6,20)$ is placed on the 9,16,1-flush, then the 6 -square must be placed on the left or there will be wasted space over the 6 -square. However, if the 6 -square is on the left, then any square placed on the 6 -square will hang over the 6 -square on the left and intersect the square over the 11-square. Therefore we cannot have $\operatorname{adj}(6,20)$ on the 9,16,1-flush. Similarly, $\operatorname{adj}(4,22)$ and $\operatorname{adj}(4,10,12)$ will yield wasted space adjacent to the 4 -square for any permutation. Thus, $e_{3}$ cannot be the 19-square.

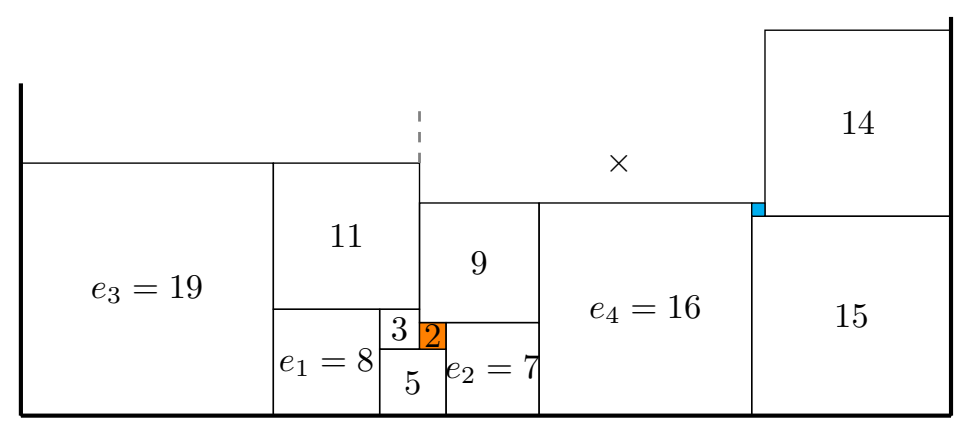

Thus, we have exhausted all cases where the set on top of the 11-square hangs over the 11-square. Therefore, we cannot have $\operatorname{adj}(2,3)$ on the 5 -square.

2. Now, place adj $(1,4)$ on the 5 -square, with the 4 -square on the left (without loss of generality). Since this is a new configuration of squares on the 5 -square, we will "reset" all $e_{i}$ squares from before. Let $e_{1}$ and $e_{2}$ be placed as before, and $e_{2}$ must be the 6 -square to avoid wasted space over the 1-square. Then the square on the right of $e_{2}$ will be greater than the 6 -square, so the set on top of the 1,6 -flush must sum to seven. The 7 -square is the only square that satisfies this sum. There are no remaining squares that sums to four, so the set of squares on the 4-square will hang over the 4-square. Therefore we need the squares adjacent to the 4,5-flush sum to nine. The 9 -square is the only square that satisfies this sum, so $e_{1}$ must be the 9 -square.

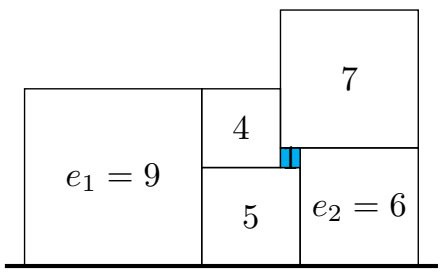


Place squares $e_{3}, e_{4}$ on the right and left of of the 6 and 9-square, respectively (note $\left|e_{3}\right|,\left|e_{4}\right| \geqslant 8$ ). If $e_{4}$ is the 8-square, then there are no remaining sets that sums to eight-so any set placed on top of the 8 -square will yield wasted space. Therefore $e_{4}$ cannot be the 8-square and so $\left|e_{4}\right| \geqslant 10$. So we need the set of squares on the 4,9 -flush to sum to 13 - this also holds if the 9-square is a corner square. But since there are no remaining sets that sum to four, then the squares placed on top of the 4-square will have a height above the 7 -square. Further, since there are no squares that sum to seven, then the set of squares on top of the 7-square will hang over the 7 -square. This implies the 6 -square cannot be a corner square. Note the set of squares that sum to 13 are $\operatorname{adj}(2,3,8), \operatorname{adj}(2,11)$, $\operatorname{adj}(3,10)$, and the 13 -square. We need two distinct sets to sum to 13 : one to go on the 4,9-flush and one to go on the 6,7 -flush.

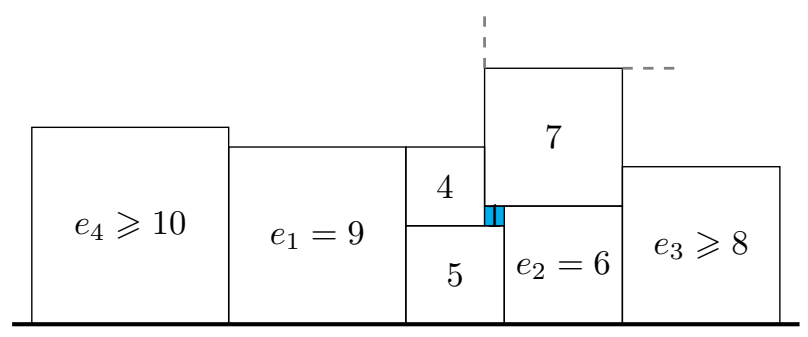

(a) Suppose $e_{3}$ is the 8 -square, place the 3 -square on top of the 8-square, and place the 2 -square on top of the 3 -square. Then any square placed next to the 3 -square will yield wasted space next to the 2 -square.

(b) Suppose $e_{3}$ is the 11-square, place the 2-square on top of $e_{3}$. Then we need the set of squares on top of the 2,7-flush to sum to nine to avoid wasted space over the 2 -square. Here there are no remaining sets that sum to nine.

(c) Suppose $e_{3}$ is the 10-square, place the 3-square on top of $e_{3}$. Then we need the set of squares on top of the 3,7-flush to sum to ten to avoid wasted space over the 3 -square. Then only set that satisfies this is $\operatorname{adj}(2,8)$. However, for either permutation, there will be wasted space above the 2-square.

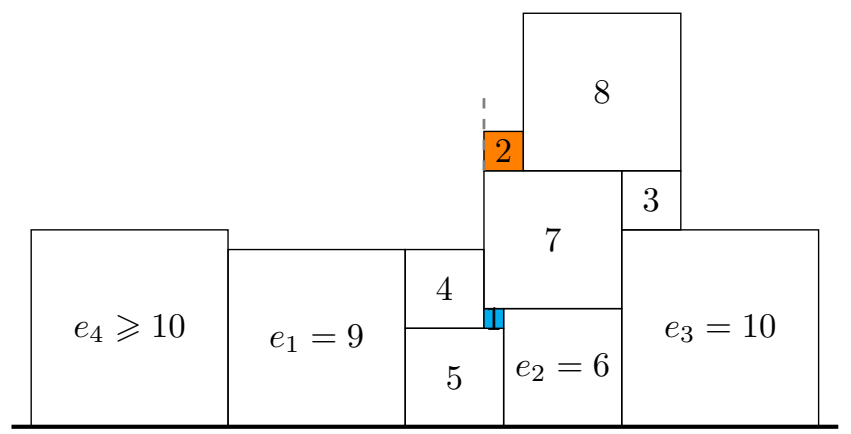

Therefore $e_{3}$ must be the 13-square.

Now, we need a set of squares that sums to 13 to be placed on the 4,9-flush. The sets are: $\operatorname{adj}(2,3,8), \operatorname{adj}(2,11)$, and $\operatorname{adj}(3,10)$. 
(a) If $\operatorname{adj}(2,3,8)$ is placed on the 4,9-flush, then the 8 -square must be placed on the right to avoid wasted space. However there will be wasted space over the 2-square for either permutation.

(b) If $\operatorname{adj}(2,11)$ is placed on the 4,9-flush, then the 2 -square must be on the left to avoid wasted space. Further the set of squares adjacent to the 2,9-flush must sum to 11 to avoid wasted space over the 2 -square and the only set that satisfies this is $\operatorname{adj}(3,8)$. However, we established earlier that $\left|e_{4}\right| \geqslant 10$. So we cannot have $\operatorname{adj}(2,11)$ on the 4,9-flush.

(c) If $\operatorname{adj}(3,10)$ is placed on the 4,9-flush, then the 3 -square must be on the right to avoid wasted space over the 3 -square. Further, $e_{4}$ must be the 12 -square to avoid wasted space over the 3 -square (there are no other sets that sum to 12 ). Note that the remaining squares less than the 10-square are the 2 and 8-square. So any squares placed on top of the 3 or 7 -square will have a height above the 10 -square. Therefore the set of squares on top of the 10-square must sum to ten. The only set that satisfies this is $\operatorname{adj}(2,8)$. However, for either permutation there will be wasted space over the 2-square. This is because the next smallest square is the 11-square and placed on either side will have a height above the 2 -square.

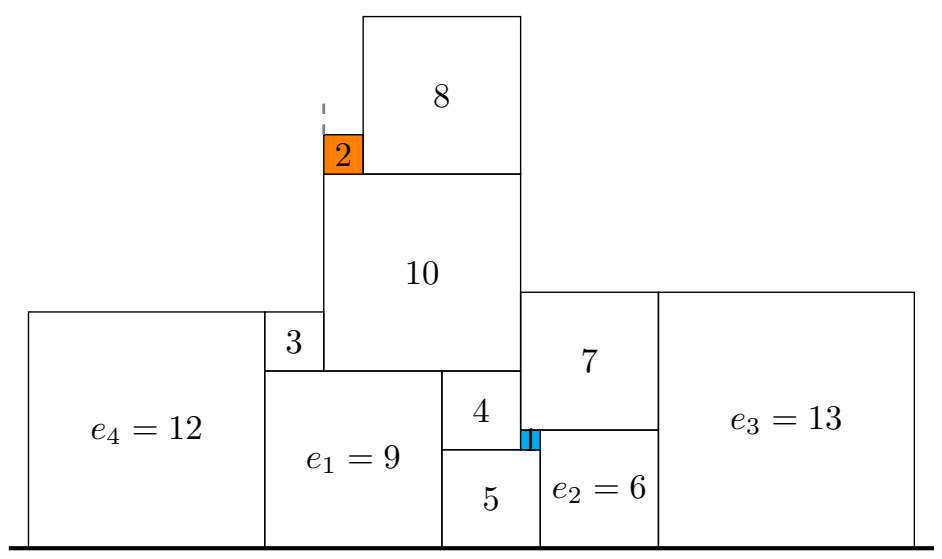

Therefore, $e_{3}$ cannot be the 13-square.

Thus, the sets $\operatorname{adj}(1,4)$ and $\operatorname{adj}(2,3)$ cannot be placed on top of the 5 -square and hence the 5 -square cannot be an edge square.

\section{Remove 6, 7, and 8 From an Edge}

Claim. We cannot have the 6, 7, and 8-square on the same edge.

Proof. Suppose the 6, 7, and 8-squares are on the edge. Then there are three cases to consider: all adjacent, one disjoint, all disjoint.

1. We consider the case where all squares are disjoint (this case will help prove the other cases). Since squares 6,7 , and 8 are disjoint on an edge, then each of them has squares $\geqslant 9$ on either side or (at most two) are 
corner squares. Therefore we need unique sets of squares to be placed on the 6,7 , and 8-square that sums to six, seven, and eight respectively. Consider the total length of these squares, namely, $6+7+8=21$, and the total length of squares $1-5$, namely, $1+2+3+4+5=15$. Since $21>15$ then there is no combination of squares $1-5$ that can uniquely be placed of squares 6,7 , and 8 . Thus, squares 6,7 , and 8 cannot be disjoint on an edge.

2. Suppose adj $(6,7,8)$. There are six different permutations of adj $(6,7,8)$. However by symmetry, we may say $\operatorname{ord}(6,7,8) \equiv \operatorname{ord}(8,7,6)$, $\operatorname{ord}(6,8,7) \equiv \operatorname{ord}(7,8,6)$, and $\operatorname{ord}(7,6,8) \equiv \operatorname{ord}(8,6,7)$. So we only need to consider three permutations.

(a) Suppose ord $(6,7,8)$ is on an edge. Then place a square on the left of the 6 -square, call this $n$; note $|n| \geqslant 9$. Then any squares placed on top of the 6 -square must sum to six; this holds if the 6-square is a corner square as well. Then we have the possible combinations: $\operatorname{adj}(1,5), \operatorname{adj}(2,4)$, and $\operatorname{adj}(1,2,3)$.

i. Suppose $\operatorname{adj}(2,4)$ is placed on top of the 6 -square. Then the set of squares placed on top of the 7 -square must sum to seven. Note that the remaining squares less than seven are the 1,3 , and 5 -squares; so there are no combination of these squares that sum to seven. This implies that there are no squares that can be placed on top of the 7-square without wasted space.

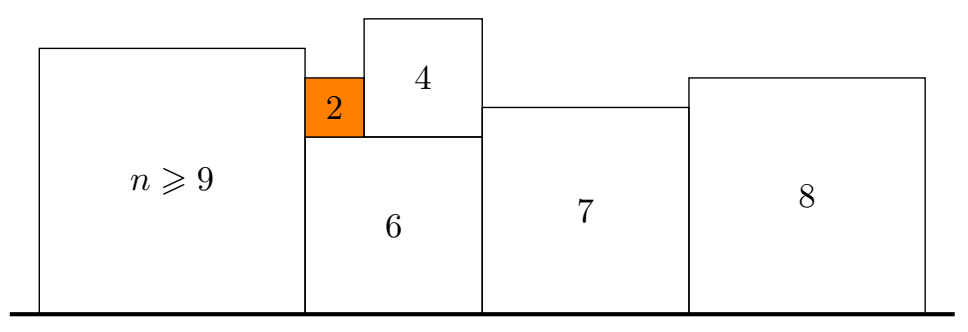

ii. Consider the two cases that involve the 1-square. If the 1-square is not on the right, then there will be wasted space over the 1-square. Place the 1-square on the right. Then, any set of squares that are placed on top of the 1,7-flush must sum to eight.

- If $\operatorname{adj}(1,2,3)$ was placed on top of the 6 -square then the remaining squares less than the 8-square are the 4 and 5 -square. Therefore there is no combination of these that sum to eight.

- If $\operatorname{adj}(1,5)$ was placed on top of the 6 -square then the remaining squares less than 8 are the 2, 3, and 4-square, so there is no combination of these that sum to eight. Therefore there will be wasted space over the 1,7-flush for any set of squares placed on the 1,7-flush.

Thus, both cases that involve the 1-square cannot be placed on top of the 6-square.

Thus we cannot have ord $(6,7,8)$ on an edge. 
(b) Suppose ord $(7,6,8)$ is on the edge. Then, any set of squares placed on top of the 6 -square must sum to six. The only combinations that satisfy that are $\operatorname{adj}(2,4), \operatorname{adj}(1,5)$, and $\operatorname{adj}(1,2,3)$.

i. Suppose $\operatorname{adj}(2,4)$ is placed on top of the 6 -square, in either permutation. Then the set of squares placed on top of the 7 -square must sum to seven. Note that the remaining squares less than the 7-square are the 1,3, and 5-squares; so there are no combination of these squares that sum to seven. Therefore, there are no squares that can be placed on top of the 7-square without wasted space.

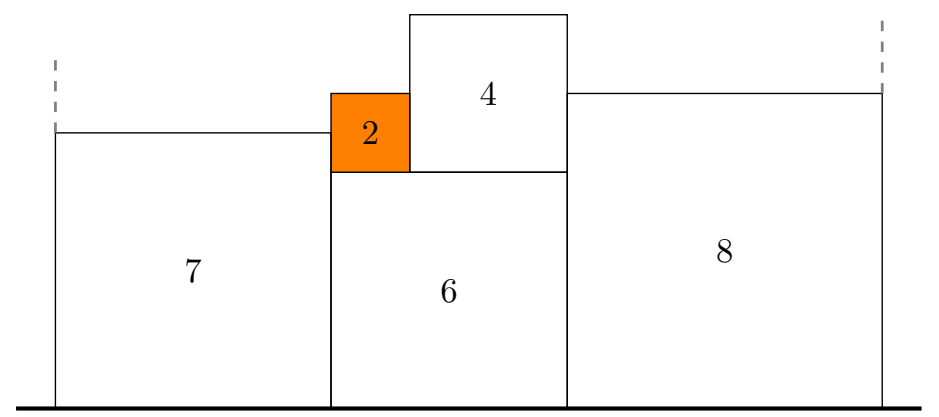

Thus, we cannot have $\operatorname{adj}(2,4)$ placed on top of the 6 -square.

ii. Consider the two cases that involve the 1-square. If the 1-square is not on the left, then there will be wasted space over the 1-square. So place the 1-square on the left. Then, any set of squares that are placed on top of the 1,7-flush must sum to eight.

- If $\operatorname{adj}(1,2,3)$ was placed on top of the 6-square then the remaining squares less than the 8-square are the 4 and 5 -square. So there is no combination of these that sum to eight.

- If $\operatorname{adj}(1,5)$ was placed on top of the 6 -square then the remaining squares less than the 8 -square are the 2,3 , and 4-square. So there is no combination of these that sum to eight.

Therefore, we cannot have $\operatorname{adj}(1,2,3)$ nor $\operatorname{adj}(1,5)$ placed on top of the 6 -square, for any permutation.

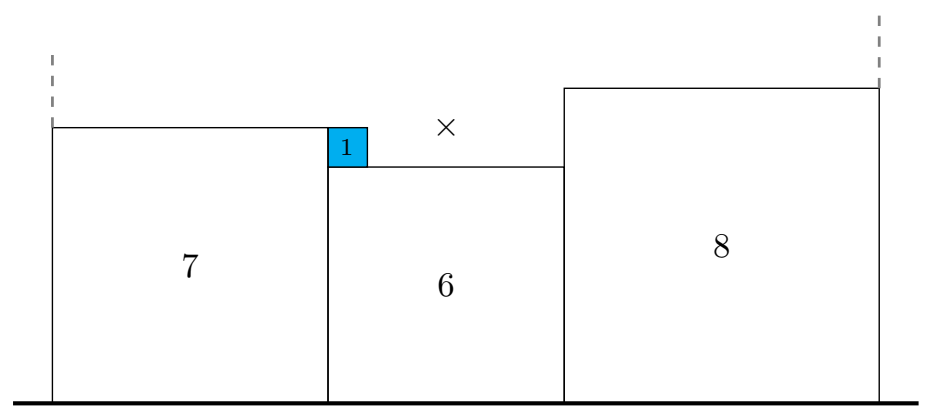

Thus, we cannot have ord $(7,6,8)$ on an edge. 
(c) Suppose ord $(6,8,7)$ is on an edge. Note that any square placed on the right of the 6 -square will be $\geqslant 9$, and similarly for the right of the 7-square. Any set of squares placed on top of the 6 and 7-square must therefore sum to six and seven respectively. However, if the 1 -square is placed on the 6-square this guarantees wasted space over the 6 -square; so place $\operatorname{adj}(2,4)$ on the 6 -square. Further, the only remaining squares less than the 7 -square are the 1,3 , and 5 -squares. Therefore there are no combinations that sum to seven, and will thus yield wasted space over the 7-square.

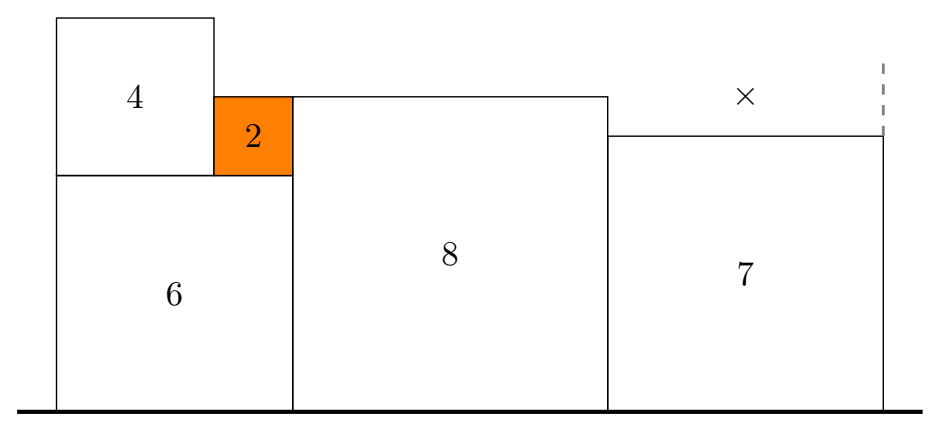

Thus, we cannot have ord $(6,8,7)$ on an edge.

We have covered the cases of $\operatorname{adj}(6,7,8)$; now we will cover the cases that involve one disjoint.

3. Suppose $\operatorname{adj}(6,7)$ with the 8 -square disjoint are all on an edge. Without loss of generality let the 6 -square be on left of the 7 -square, and place the 8 -square placed arbitrarily on the base disjoint from $\operatorname{adj}(6,7)$. Note that either the 6 or 7 -square is a corner square, or the squares placed on either side of $\operatorname{adj}(6,7)$ is $\geqslant 9$; this holds for the 8 -square as well.

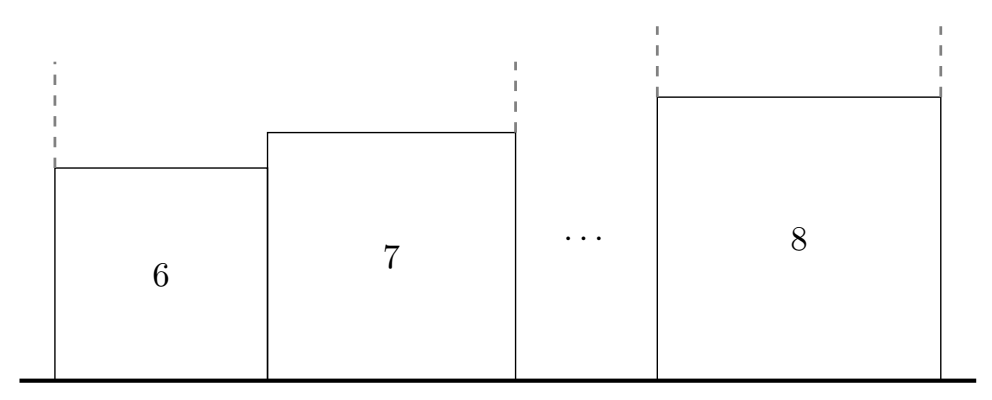

Note that we must have unique sets of squares to be placed on the 6 and 8 -squares that sum to six and eight respectively. The sets of squares that sum to six are $\operatorname{adj}(2,4), \operatorname{adj}(1,5)$, and $\operatorname{adj}(1,2,3)$. 
(a) Suppose $\operatorname{adj}(2,4)$ is placed on top of the 6 -square. Then we need unique sets of squares to be placed on the 7 and 8 -square. That is, we need unique sets of squares on the 6,7 , and 8-square, which cannot happen by the all disjoint case.

(b) Consider the two cases that involve the 1-square. If the 1-square is not on the right, then there will be wasted space over the 1-square. So place the 1-square on the right. Then, any set of squares that are placed on top of the 1,7-flush must sum to eight; $\operatorname{adj}(3,5)$ is the only set satisfying this sum. However this leaves no remaining sets to be placed on the 8-square.

Thus, we cannot have $\operatorname{adj}(6,7)$ with the 8 -square disjoint.

4. Suppose $\operatorname{adj}(6,8)$ with the 7 -square disjoint are all on an edge. Without loss of generality let the 6-square be on left of the 8-square, and place the 7 -square placed arbitrarily on the base disjoint from $\operatorname{adj}(6,8)$. Note that either the 6 or 8 -square is a corner square, or the squares placed on either side of $\operatorname{adj}(6,8)$ is $\geqslant 9$; this holds for the 7 -square as well.

Then any set of squares placed on top of the 6-square must sum to six. The possible combinations of squares that sum to six $\operatorname{are} \operatorname{adj}(1,2,3), \operatorname{adj}(1,5)$, and $\operatorname{adj}(2,4)$. However if the 1 -square is placed on the 6 -square, then there will be wasted space for any permutation of any set placed on the remainder of the 6 -square; so place adj $(2,4)$ on the 6 -square. Note any set of squares placed on top of the 7 -square must sum to seven. The only remaining squares less than the 7 -square are the 1,3 , and 5 -square. Therefore there are no remaining sets that sum to seven and will yield wasted space over the 7 -square.

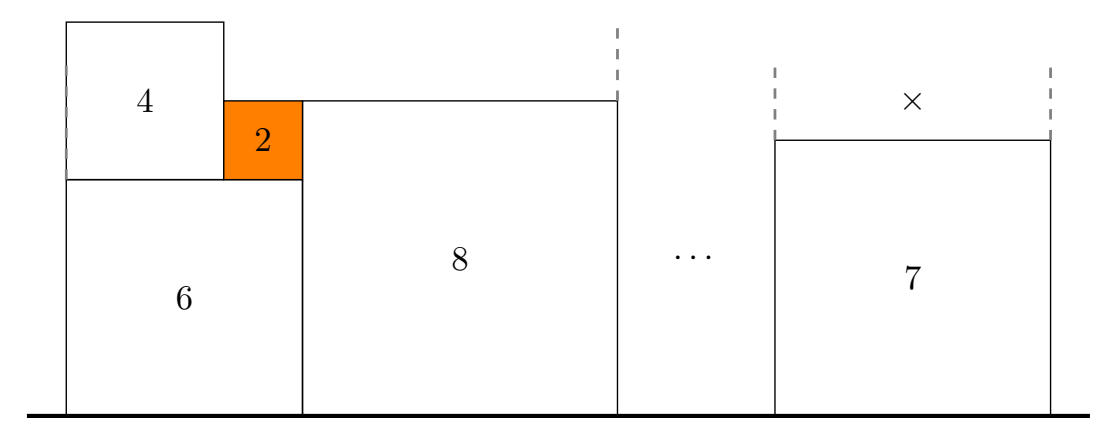

Thus, we cannot have $\operatorname{adj}(6,8)$ with the 7 -square disjoint.

5. Suppose $\operatorname{adj}(7,8)$ with the 6 -square disjoint are all on the edge. Without loss of generality let the 6-square be on left of the 8-square, and place the 7 -square placed arbitrarily on the base disjoint from $\operatorname{adj}(6,8)$. Either the 7 or 8 -square is a corner square, or the squares placed on either side of $\operatorname{adj}(7,8)$ is $\geqslant 9$; this holds for the 6 -square as well. 


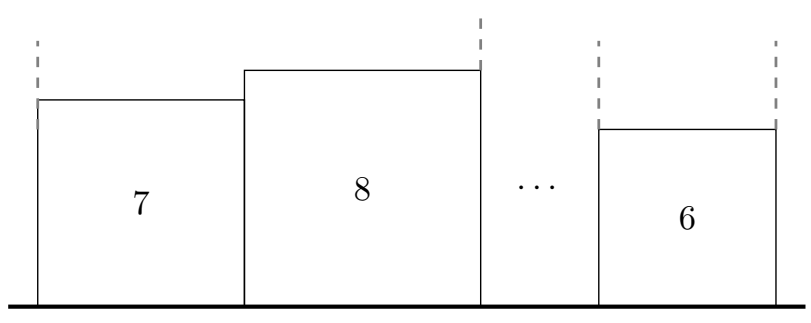

We must have the set of squares placed on top of the 7-square sum to seven, and similarly, the set of squares on the 6-square must sum to six. The only unique sets that satisfy this are: $\operatorname{adj}(1,5)$ on the 6 -square and $\operatorname{adj}(3,4)$ on the 7 -square. By placing adj $(3,4)$ on the 7 -square, the set of squares on top of the 8-square must then sum to eight. But the 2-square is the only remaining square less than the 8 -square. Thus, there will be wasted space over the 8 -square. Thus, we cannot have $\operatorname{adj}(7,8)$ with the 6-square disjoint.

Therefore, all cases of the 6, 7, and 8-square placed on the edge have been eliminated.

\section{Proof of Theorem 1(iii)}

Proof. Suppose the 6 -square is on the edge but not adj(6,7). Then either the 7 -square is on the same edge but disjoint or the 7 -square is not on that edge. Consider the case when the 6 and 7 -squares are on an edge but disjoint. This case yields wasted space since it is logically equivalent to having adj $(6,8)$ and 7 -square disjoint or having $(6,8,7)$ on an edge.

Suppose the 7-square is not on an edge. Then the 6 -square will have squares on either side which are $\geqslant 8$ (note the 6 -square cannot be a corner square by Lemma 1). If the 8 -square is not adjacent to the 6 -square then we have $\operatorname{ord}\left(n_{1}, 6, n_{2}\right)$ but $\left|n_{1}\right|,\left|n_{2}\right|>8$ which guarantees wasted space over the 6 -square. So we must have $\operatorname{adj}(6,8)$. Furthermore, if the 1 -square is placed on top of the 6 -square, there will be wasted space. Then we must have $\operatorname{adj}(6,8)$ on an edge and $\operatorname{adj}(2,4)$ on the 6 -square. Moreover the 2 -square must be placed adjacent to the 8 -square to avoid wasted space. Now we need a set of squares that sum to ten to be placed on top of the 2,8-flush. The remaining sets that sum to ten are $\operatorname{adj}(1,9)$, $\operatorname{adj}(3,7)$, and the 10 -square.

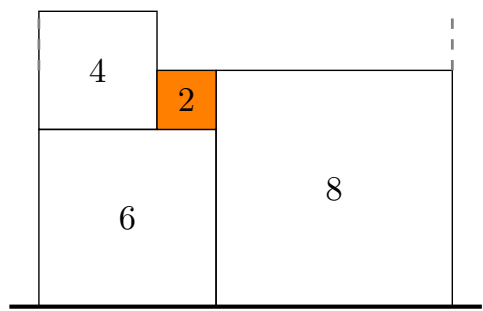

We begin by considering when $\operatorname{adj}(1,3)$ is placed on the 4 -square. Then the 10 -square is the only set that sums to ten. So the 10 -square must be placed on the 2,8-flush. Then any square placed on the 3 -square will hang over the 
3 -square and yield wasted space over the 1 -square. Thus adj(1,3) cannot be placed on the 4-square. Thus, any set of squares on the 4-square will hang over the 4-square on the left (since any square placed on the 2-square will have a height above the 4-square). That is, we need two unique sets of squares that sum to ten. Note that we cannot have $\operatorname{adj}(3,7)$ on the left of the 4,6 -flush by assumption (this would be $\operatorname{adj}(6,7)$ ).

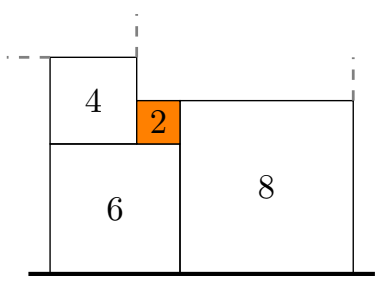

1. Suppose the $\operatorname{adj}(6,9)$ is on the edge, with the 1 -square on the 9-square. Then the 5-square must be placed on the 1,4-flush, otherwise there will be wasted space next to the 1-square. If there is a square adjacent to the 9 -square on the edge, it will be $\geqslant 10$. This implies that the remaining set of squares on the 9-square must sum to eight (this logically equivalent if the 9 -square is a corner square as well). However, there are no remaining sets of squares that sum to eight. Thus we cannot have $\operatorname{adj}(1,9)$ on the left of the 4,6-flush.

2. Suppose $\operatorname{adj}(6,10)$ is on the edge, and $\operatorname{adj}(1,9)$ is placed on the 2,8-flush. Then the 1-square must be placed on the right to avoid wasted space. Then any square placed on the 1-square would hang over to the right of the 1-square. (This implies the 8-square is not a corner since it would otherwise be a contradiction.) So, we need a set of squares that sums to nine to be placed adjacent to the 1,8-flush. However, there are no remaining sets that sum to nine. Thus we cannot have $\operatorname{adj}(1,9)$ on the 2,8-flush.

3. Suppose $\operatorname{adj}(6,10)$ is on the edge, and $\operatorname{adj}(3,7)$ is placed on the 2,8 -flush. If the 3 -square is placed on the left then any set of squares placed on the 3 -square will hang over the 3-square and yield wasted space next to the 3 -square. So place the 3-square on the right. Again, any set of squares placed on the 3-square will hang over the 3-square, which implies the 8 -square is not a corner square. Note the 11-square is the only remaining set of squares that sums to eleven and therefore we must have adj $(8,11)$ on the edge.

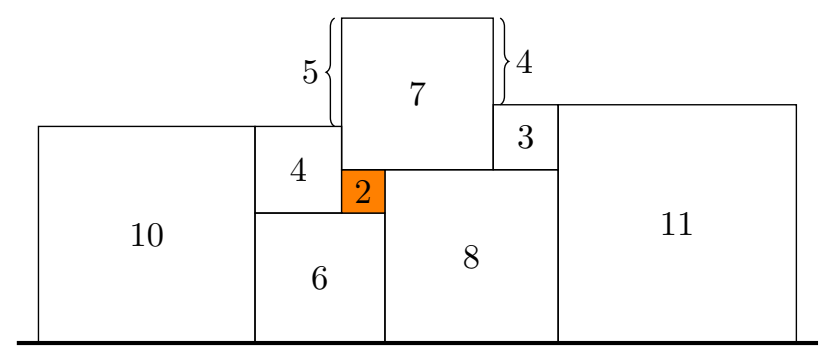


Here we see that any square placed on the 3-square will have a height above the 7 -square. Further, any set of squares placed on the 7 -square will hang over the 7-square (on the left). Therefore the 5 -square is the only square that can be placed on the on the 4-square without wasted space. Now, there are no remaining sets of squares that sum to five, so the set of squares on the 5,7 -flush must sum to 12 . The 12 square is the only square that satisfies this sum, so place the 12 -square on the 5,7 -flush.

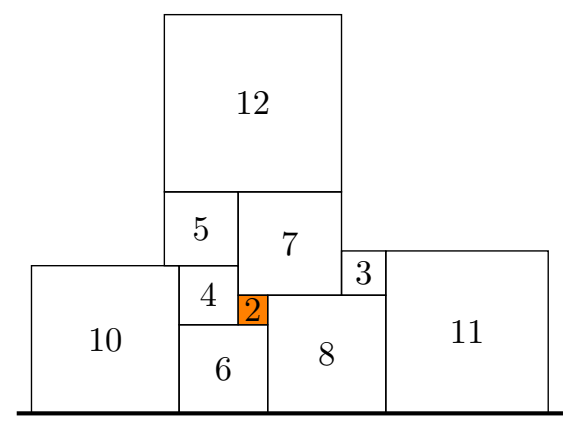

Suppose the 10-square is a corner square. Then the remaining squares on the 4,10-flush must sum to nine. The 9-square is the only remaining square satisfying this sum. However, since the 9-square is the only remaining set, then there will be wasted space for any set of squares placed on the 9-square. Thus, the 10-square is not a corner square.

Suppose the 9 -square is placed on the edge adjacent to the 10 -square. Then the set of squares on the 9-square must sum to nine. But there are no remaining sets that satisfy this, therefore there must be a square $\geqslant 13$ adjacent to the 10-square on the edge. A similar argument holds for the 11-square; that is, if the 11-square is not a corner square, there must be a square $\geqslant 13$ to the right of the 11-square.

We have just determined that the 10 -square is not a corner square but is adjacent to a square that is at least the 13-square. Therefore the set of squares on the 10-square must sum to nine, so place the 9 -square on the 10 -square. Then any set of squares placed on the 9 -square will hang over the 9 -square. So we need the set of squares on the 9,10-flush to sum to 19 . The only sets that satisfy this are $\operatorname{adj}(1,18)$ and the 19 -square. However, if $\operatorname{adj}(1,18)$ is placed on the 9,10 -flush then any set of squares placed on the 1,9-flush would yield wasted space next to the 1-square. Therefore we place the 19-square adjacent to the 10-square on the edge.

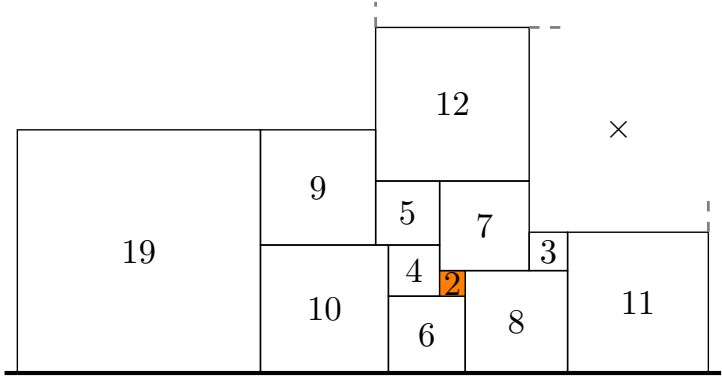


Note that squares $2-12$ have been used. So any square placed on the 9 -square will have a height above the 12-square. Similarly any set of squares placed on the 12-square will hang over the 12-square. Thus the remaining set of squares adjacent to the 7,12-flush must sum to 16 . The only sets that sum to 16 is $\operatorname{adj}(1,15)$ and the 16 -square. However since the set of squares on the 3,11-flush must sum to 14 then this is a contradiction for both cases.

Thus, we cannot have $\operatorname{adj}(3,7)$ on the 2,8 -flush.

4. Suppose the 10-square is placed on the 2,8-flush. Consider when adj(1,3) is placed on the 4-square. Then the 1-square must be on the left to avoid wasted space. Then any set of squares placed on the 4-square will hang over the 4-square and therefore yield wasted space over the 1-square. Therefore $\operatorname{adj}(1,3)$ cannot be on the 4 -square. Further this implies any set of squares placed on the 4 -square will hang over the 4 -square. So we need a set squares that sums to ten to be placed adjacent to the 4,6-flush. The only remaining set that satisfies this is adj(1,9) (recall the 7 -square cannot be on the edge by assumption). Place the 9 -square on the edge to the left of the 6 -square, and the 1-square on top of the 9 -square. Then the 5 -square must be placed on the 1,4-flush to avoid wasted space on the left of the 1-square. Note any set of squares placed on the 5-square will hang over the 5-square. However, there are no remaining sets of squares that sum to six to be placed adjacent to the 1,5-flush. Thus, we cannot have the 10-square placed on the 2,8-flush.

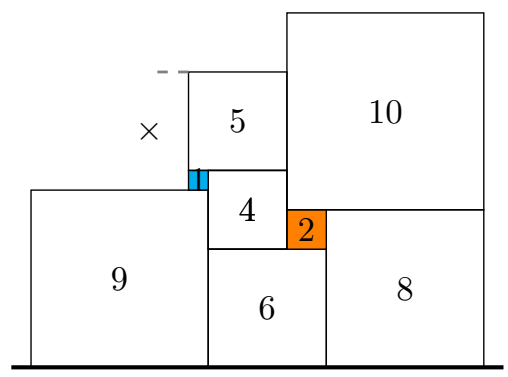

Therefore, we have exhausted all cases with the 7 -square both on an edge (disjoint from the 6-square) and the 7 -square not on an edge. Thus, we conclude that if the 6 -square is on an edge, then we must have $\operatorname{adj}(6,7)$ on an edge.

\section{Proof of Theorem 1/(iv)}

Suppose $\operatorname{ord}(a, n, b) \subseteq \epsilon$ is an ordered subset of a packing $\mathcal{P}$, where $n$ is the smallest square lying on $\epsilon$. We will show that

$$
\min \{|a|,|b|\} \leqslant 2|n|-1
$$

To begin, we need a preliminary lemma which will reduce the number of cases to consider. 
Lemma 2. Suppose $\operatorname{ord}(a, n, b)$ is as above, and let $\Gamma=\left\{a_{1}, a_{2}, \ldots, a_{k}\right\}$ be a partition of $|n|$ into distinct integers; that is, $|n|=a_{1}+\cdots+a_{k}$ and $a_{i} \neq a_{j}$ for $i \neq j$. Furthermore, suppose that the corresponding $\operatorname{squares} \operatorname{adj}\left(a_{1}, \ldots, a_{k}\right)$, where $\left|a_{i}\right|<\left|a_{i+1}\right|$, all lie on top of $n$ in $\mathcal{P}$.

1. If $|a|,|b| \geqslant|n|+2$ then $1 \notin \Gamma$.

2. If $|a|,|b| \geqslant|n|+3$, then $2 \notin \Gamma$.

3. If $|a|,|b| \geqslant|n|+6$, then $3 \notin \Gamma$.

Proof. Suppose $1 \in \Gamma$ and $|a|,|b| \geqslant|n|+2$. Since $|a|,|b| \geqslant|n|+2$, then this guarantees wasted space over the 1 -square, for any permutation of $\operatorname{adj}\left(a_{1}, \ldots, a_{k}\right)$. A similar argument holds (2).

Now, suppose $|a|,|b| \geqslant|n|+6$ and $3=a_{1} \in \Gamma$ for contradiction. Then we have $\Gamma=\left\{3, a_{2}, \ldots, a_{k}\right\}$ where $a_{i}>3$ for each $i=2, \ldots, k$. We must have a set of squares on the 3 -square that sums to three. $\operatorname{Adj}(1,2)$ is the only set satisfying this sum; place $\operatorname{adj}(1,2)$ on the 3 -square. Let $\operatorname{adj}\left(3, a_{2}\right)$, if $\left|a_{2}\right|>4$ then this guarantees wasted space over the 1-square for either permutation. Therefore let $a_{2}$ be the 4 -square and makes a flush with the 1 -square. Then the 5 -square is the only square that can be placed on the 1,4-flush. However, this will guarantee wasted space over the 2-square.

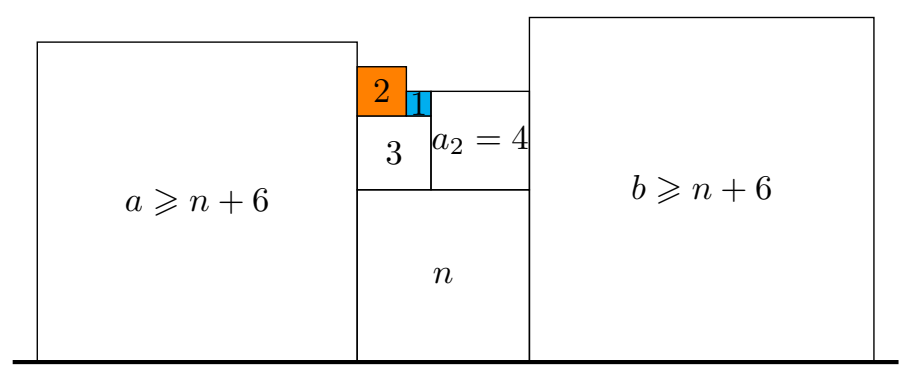

Note that if $|n|>7$ then we can improve the restriction of (3) to: $|a|,|b| \geqslant|n|+5$, then $3 \notin \Gamma$.

Example: Consider when $n$ is the 7 -square and the smallest square on an edge, $\epsilon$. Further suppose that ord $(10,7,9)$ in on $\epsilon$. We need to determine the sets of squares that sum to seven: the distinct partitions of seven are $1+6,2+5,3+4$, and $1+2+4$. By Lemma 2, we can remove the cases that use the 1-square; that is, the remaining partitions are $2+5$ and $3+4$. So the remaining sets of squares that can be placed on the 7 -square are $\operatorname{adj}(2,5)$ and $\operatorname{adj}(3,4)$.

Proof. (of Theorem 1.1 (iv)). Suppose $n$ is the smallest square on the edge. We want to show that we cannot have the second smallest square be greater than or equal to $2 n-1$. Recall the 6 -square is the smallest square we can have on the edge. Further, note that there must be a minimum of four squares on the edge. So the maximum that $n$ can be is the 10 -square, otherwise if $n$ were the 11-square, then the smallest set of squares is $(11,21,22,23)$ which sum is greater that 70 . This leaves us with five cases to check: $n=6,7,8,9$, and 10 . 
1. Suppose $n$ is the 6 -square. Since the 6 -square is on an edge, then by Theorem 1](iii) we must have $\operatorname{adj}(6,7)$ on the edge, and this is case holds.

2. Suppose $n$ is the 7 -square, let $a$ be the 13 -square and $b$ such that $|b| \geqslant 14$ on the right and left (without loss of generality) of the 7-square respectively. Then the only squares that can be placed on top of the 7 -square are $\operatorname{adj}(1,6), \operatorname{adj}(2,5), \operatorname{adj}(3,4)$, and $\operatorname{adj}(1,2,4)$. These are all ruled out by Lemma 2.

3. Suppose $n$ is the 8 -square where $a$ is the 15 -square placed on the right and $b$ such that $|b| \geqslant 16$ on the left (without loss of generality). The only squares we have that can go on the 8-square are $\operatorname{adj}(1,7), \operatorname{adj}(2,6)$, $\operatorname{adj}(3,5), \operatorname{adj}(1,2,5)$, and $\operatorname{adj}(1,3,4)$. However, by Lemma 2 these are all ruled out.

4. Suppose $n$ is the 9 -square where $a$ is the 17 -square placed on the right and $b$ such that $|b| \geqslant 18$ on the left (without loss of generality). We have the following possibilities that can go on the 9-square are $\operatorname{adj}(1,8)$, $\operatorname{adj}(2,7), \operatorname{adj}(3,6), \operatorname{adj}(4,5), \operatorname{adj}(1,2,6), \operatorname{adj}(1,3,5), \operatorname{and} \operatorname{adj}(2,3,4)$. However, by Lemma 2 we have only to check $\operatorname{adj}(4,5)$.

Suppose $\operatorname{adj}(4,5)$ is placed on top of the 9-square with the 4-square on the left. The only squares that can be placed on the 4-square are the 1 , 2 , and 3 -squares. Placing the 2-square on top of the 4-square will yield wasted space. Place adj $(1,3)$ on top of the 4 -square with the 3 -square on the left (otherwise there is wasted space over the 1-square). Notice, we have a 1,5-flush, and the set of squares placed on the 1,5-flush must sum to six. The 6 -square is the only remaining square that satisfies this sum; so place the 6 -square on the 1,5-flush. The only square that can placed on top of the 3 -square is the 2 -square, and that would yield wasted space on the left/ right of the 2-square.

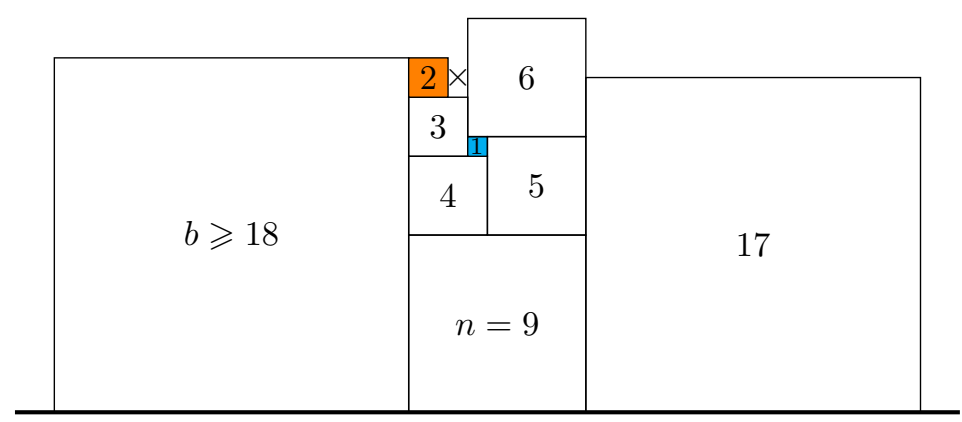

5. Suppose $n$ is the 10 -square where $a$ is the 19 -square placed on the right and $b$ such that $|b| \geqslant 20$ on the left (without loss of generality). Then the possible sets of squares that can go on the 10-square are $\operatorname{adj}(1,9), \operatorname{adj}(2,8)$, $\operatorname{adj}(3,7), \operatorname{adj}(4,6), \operatorname{adj}(1,2,7), \operatorname{adj}(1,3,6)$, and $\operatorname{adj}(2,3,5)$. Again applying Lemma 2 we are only left with the case $\operatorname{adj}(4,6)$.

Suppose $\operatorname{adj}(4,6)$ is placed on top of the 10-square; consider when the 6 -square is on the right. Then, the only square that can be placed on the 4 -square without wasted space is adj $(1,3)$. However, this yields wasted space over the 1-square for either permutation. 


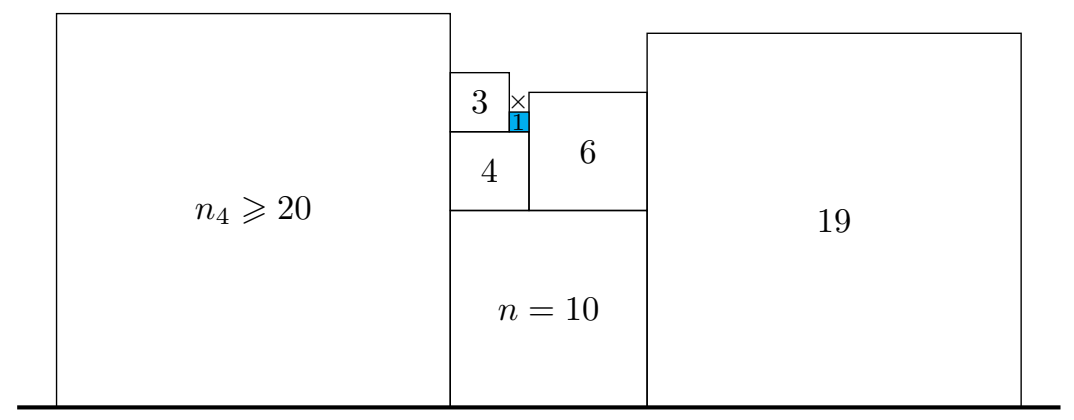

Therefore, with the constraints of the $70 \times 70$ square, we cannot have $n$ and $2 n-1$ be the smallest squares on the edge.

\section{Proof of Theorem 2}

Proof. Suppose $v=\{9,14,23,24\}$ is an edge. The 9-square must be either a corner square or a non-corner square.

1. Suppose the 9 -square is a corner square. Then we must have $\operatorname{adj}(9,14)$ by Theorem 1/(iv)] Further, the square on the adjacent edge (i.e., $c$ or $c_{o p}$ ) that is adjacent to the 9 -square must be the 6,7 , or 8 -square. Similar to the proof of Lemma 2, we are guaranteed wasted space for the 9-square adjacent to the 6,7 , and 8-squares.

2. Suppose the 9-square is not a corner square. Again by Theorem 1](iv) we must have $\operatorname{adj}(9,14)$. Note that we also have either $\operatorname{adj}(9,23)$ or $\operatorname{adj}(9,24)$. Then by Lemma $2 \operatorname{adj}(4,5)$ is the only set of squares that can be placed on the 9 -square. Further, adj(1,3) will be the only squares that can be placed on the 4-square, without wasted space. However, any set of squares placed on the 3 -square will hang over the 3 -square. The 2 -square is the only square that can be placed under the overhang from the 3 -square. However, any set of squares placed on the 2,3-flush will hang over the 2 -square, which guarantees wasted space. Thus, the 9 -square must be a corner; hence a contradiction.

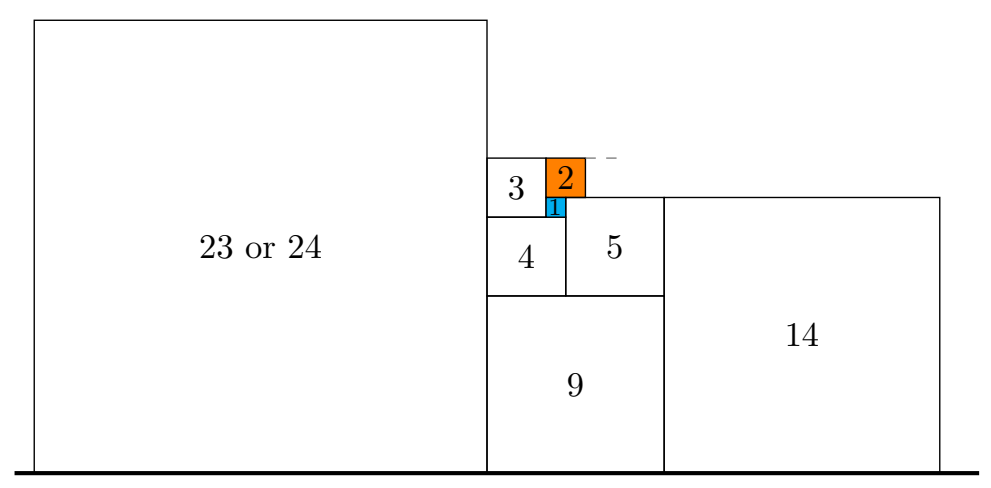

Therefore combining these cases, we conclude $v=\{9,14,23,24\}$ is not an edge. Thus establishing the theorem. 


\section{Constructing the Frame}

To construct a potential packing $\mathcal{P}$, we first need to determine the edges. In this section, we present the MATLAB code that generates all possible frames.

\section{The Matrix A}

Here we determine the matrix $A$ : all possible combinations of squares that sum to 70 taking Theorem 1/(i) into account. If we do not include these restraints initially, then MATLAB cannot handle the calculation.

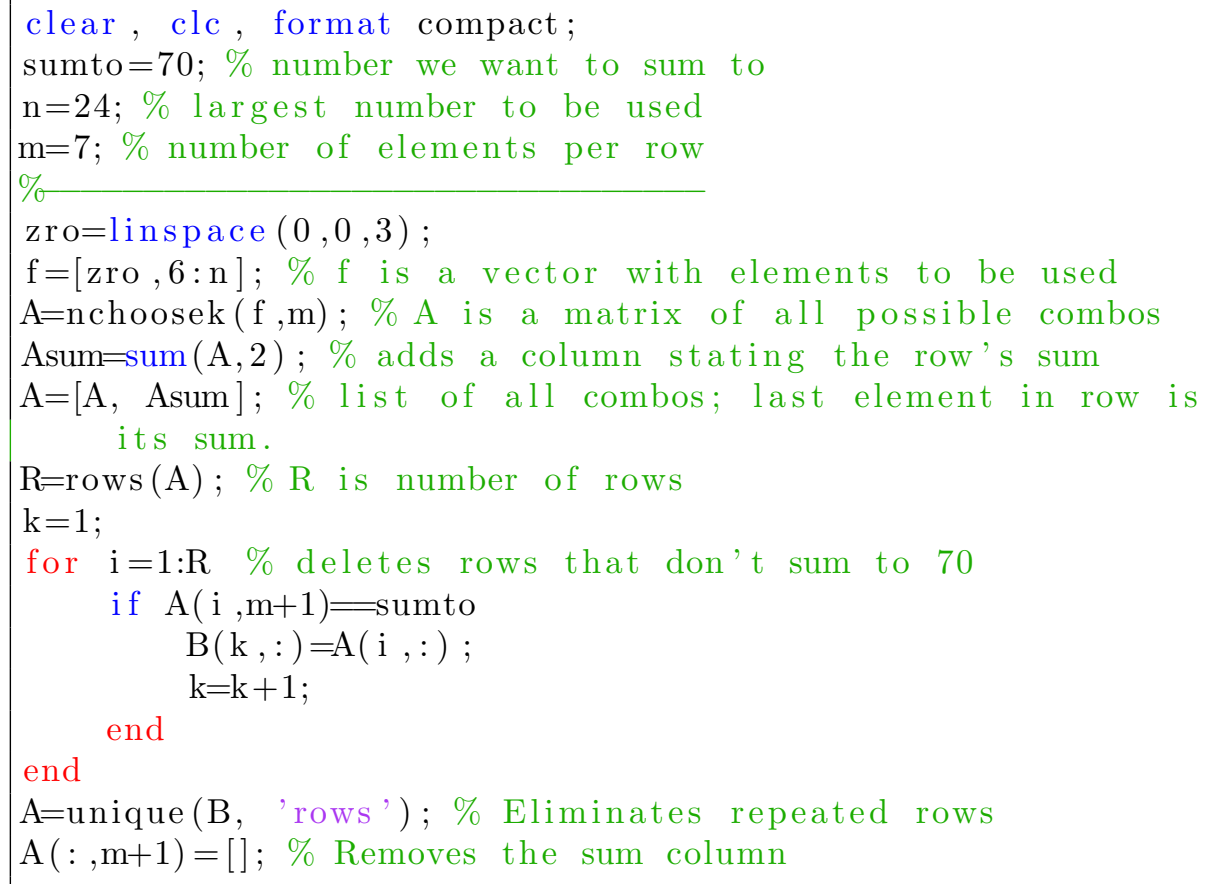

The purpose of the first line is to keep the display cleaner. In lines $2-4$ we define the variables sumto, $n, m$ where sumto $:=70$ since we want integer numbers that sum to $70, n:=24$ since we use the numbers $1-24$, and $m:=7$ since there will be at most seven squares on an edge (due to the restraints stated earlier). In line 6 , we have linspace $(0,0,3)$ which creates a vector with three elements "from zero to zero" (i.e., a vector with three zeros only). The number of zeroes is the max possible squares on an edge minus the minimum possible squares on an edge. The zeros act as placeholders for the matrices computed. On line 7 we make a vector $f=[z r o, 6: n]$ which means it has the elements from zro and $6-24$ (recall $n:=24$ ). On line 8 we create the matrix $A$ using the nchoosek function, which creates all possible combinations of the elements in $f$ using $m:=7$ columns. Lines 9 and 10 create an eighth column in the matrix $A$ which is the sum of each of the rows accordingly. In lines 11 and 12 , we create parameters for the loop which begins on line 13: $R$ is the number of rows in $A$ 
and $k$ will be a counter in the loop. The for loop removes every row that does not have 70 as the eighth element - since the last column is the sum of each row. Finally, line 19 removes any repetition that may have occurred and line 20 removes the entire eighth column - it is no longer required and removing it will reduce later computation.

Next, the remaining constraints from Theorem 1 are included. In particular, we eliminate rows that do not satisfy at least one Theorem 1[(ii) (v). Using the same $R=\operatorname{rows}(A)$ from above, we run another for loop.

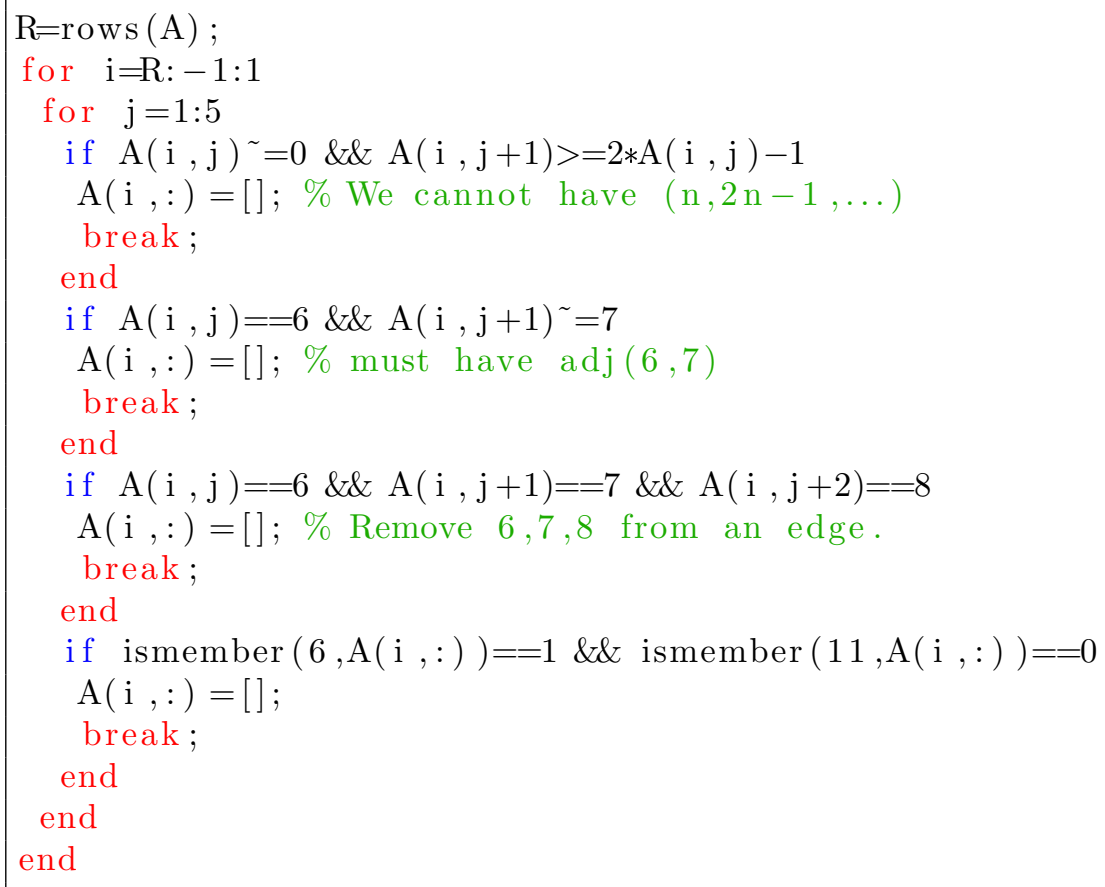

Each if-statement is a different restriction. MATLAB performs this by removing the cases that do not satisfy the claim; e.g., if there is a row in $A$ that has $(6,9, \ldots)$ then that is removed since it does not satisfy Theorem 1(iii). Therefore from here on, $A$ is the matrix of all possible ways to sum to 70 given our restraints from Theorem 1. (Note that we use a for-loop where $i: R \rightarrow 1$. It was later determined that a while loop is more easily manageable and yields the same result. This is why we use a for-loop first but while-loops later.)

\section{The Matrix $\mathbf{A}_{\mathrm{op}}$}

Next, we fix a row $v \in A$ and remove all rows which have no common element with $v$ (not including zeros). 


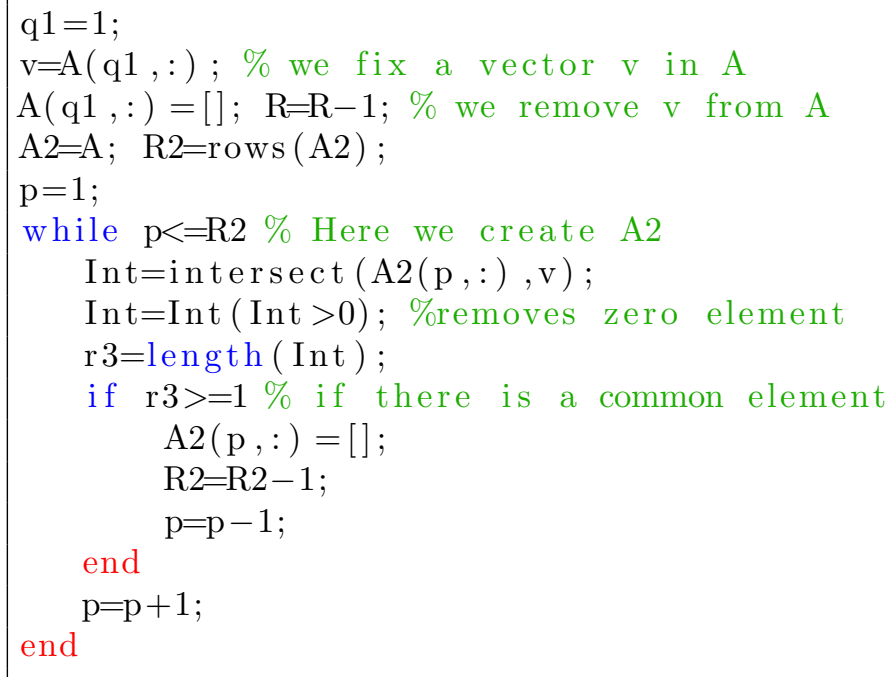

Note that line 1 states $q 1=1$; this is for future potential loops. By adding this we can later add a while loop to loop through all different choices of $v$, but for now it determines our fixed $v$.

\section{The Matrix $\mathrm{C}$}

Next, we fix a row $v_{o p} \in A_{o p}$ and create the matrix $C$ which each row has one common element with $v$ and $v_{o p}$. This will also done for the other fixed edges.

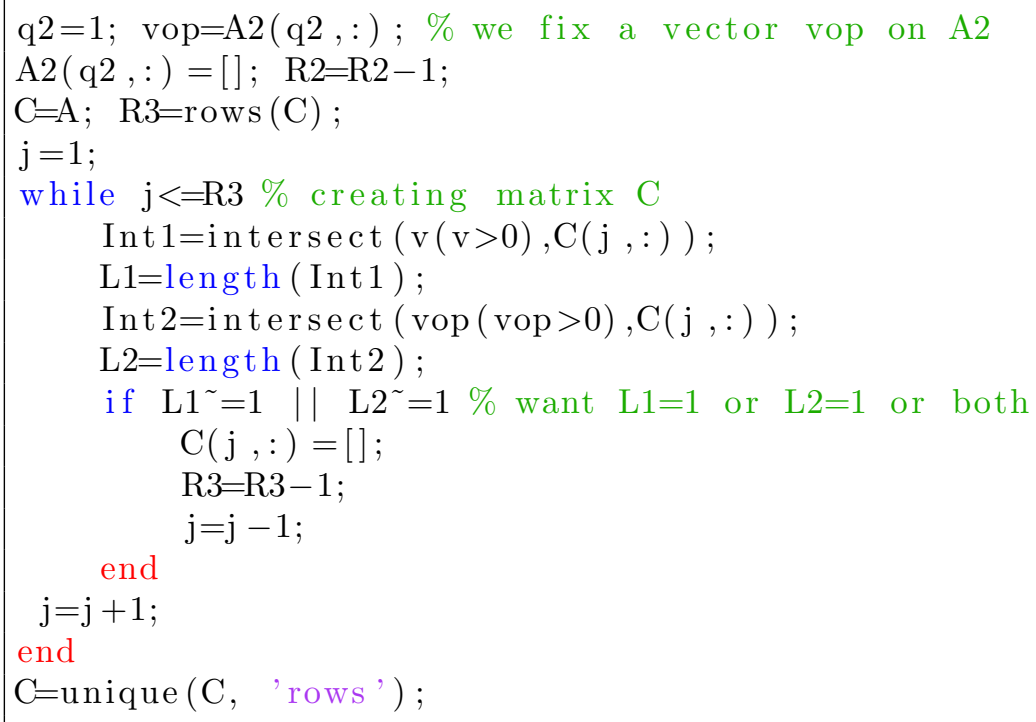

The matrix $C$ is now the matrix where each row corresponds to an edge of $\mathcal{P}$ such that there is exactly one square in common with $v$ and one (different) square in common with $v_{o p}$. 
Throughout the following theorems, let $\mathcal{P}$ be a packing with edges $\left\{\epsilon_{i}\right\}$. Define $a_{i}=\min \epsilon_{i}$ to be the smallest square on each edge, and without loss of generality assume $\left|a_{i}\right|<\left|a_{i+1}\right|$. Let

$$
S=\left\{1,2, \ldots, a_{4}\right\}-\left\{a_{1}, a_{2}, a_{3}, a_{4}\right\} \subseteq \mathcal{P}
$$

be the set of all possible squares that could be placed on top of all $a_{i}$-squares.

Theorem 4. If $\mathcal{P}$ is a packing, then $\mathcal{P}$ must satisfy

$$
\sum_{i=1}^{4}\left|a_{i}\right| \leqslant \sum_{n \in S}|n| .
$$

Proof. Consider a packing $\mathcal{P}$ such that

$$
\sum_{i=1}^{4}\left|a_{i}\right|>\sum_{n \in S}|n|
$$

for contradiction. Then we can place the squares $\operatorname{adj}(1,2, \ldots, \max \{S\})$ such that there is a common flush. Similarly, on the opposing side of the flush place $\operatorname{adj}\left(a_{1}, a_{2}, a_{3}, a_{4}\right)$. By assumption $|\operatorname{adj}(1,2, \ldots, \max \{S\})|<\left|\operatorname{adj}\left(a_{1}, a_{2}, a_{3}, a_{4}\right)\right|$, so any permutation of either sets of squares shows that there will be wasted space over $a_{i}$ for some $i=1,2,3,4$. Therefore a packing $\mathcal{P}$ must satisfy

$$
\sum_{i=1}^{4}\left|a_{i}\right| \leqslant \sum_{n \in S}|n|,
$$

as needed.

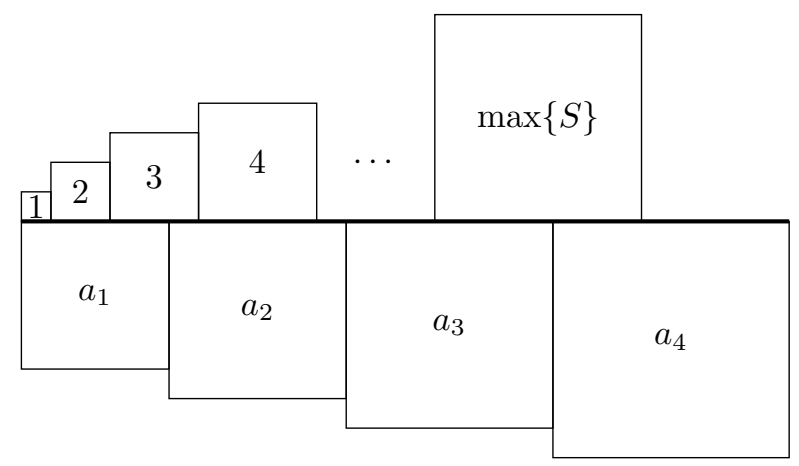

\section{Example Let}

$$
\partial \mathcal{P}=\{9,14,23,24\} \cup\{10,17,21,22\} \cup\{11,13,22,24\} \cup\{12,17,18,23\}
$$

be the set of all edge squares of a packing $\mathcal{P}$. The smallest squares on each edge are $\{9,10,11,12\}$. Then $S=\{1, \ldots, 8\}$, so we have $\sum_{n \in S}|n|=1+\cdots+8=36$.

Further, $\sum\left|a_{i}\right|=9+10+11+12=42$. That is,

$$
\sum_{n \in S}|n|=36<42=\sum\left|a_{i}\right| .
$$

Thus by Theorem 4, $\mathcal{P}$ will yield wasted space. 
Corollary 1. If $\mathcal{P}$ is a packing, then $\mathcal{P}$ must satisfy

$$
4\left(\left|a_{1}\right|+\left|a_{2}\right|+\left|a_{3}\right|+\left|a_{4}\right|\right) \leqslant\left|a_{4}\right|\left(\left|a_{4}\right|+1\right) .
$$

Proof. Consider the inequality of Theorem 4 and recall $\left|a_{i}\right|<\left|a_{i+1}\right|$. Then,

$$
\begin{gathered}
\sum_{i=1}^{4}\left|a_{i}\right| \leqslant \sum_{n \in S}|n| \\
\left|a_{1}\right|+\left|a_{2}\right|+\left|a_{3}\right|+\left|a_{4}\right| \leqslant\left(1+\cdots+\left|a_{4}\right|\right)-\left(\left|a_{1}\right|+\left|a_{2}\right|+\left|a_{3}\right|+\left|a_{4}\right|\right) \\
2\left(\left|a_{1}\right|+\left|a_{2}\right|+\left|a_{3}\right|+\left|a_{4}\right|\right) \leqslant 1+\cdots+\left|a_{4}\right| \\
2\left(\left|a_{1}\right|+\left|a_{2}\right|+\left|a_{3}\right|+\left|a_{4}\right|\right) \leqslant \frac{\left|a_{4}\right|\left(\left|a_{4}\right|+1\right)}{2} \\
4\left(\left|a_{1}\right|+\left|a_{2}\right|+\left|a_{3}\right|+\left|a_{4}\right|\right) \leqslant\left|a_{4}\right|\left(\left|a_{4}\right|+1\right),
\end{gathered}
$$

as needed.

In MATLAB it is more practical to use Corollary 1 as opposed to Theorem 4

Example Let $\partial \mathcal{P}$ be as in the above example. We can see that

$$
4(9+10+11+12)=4(42)=168>156=12(13) .
$$

So, by Corollary 1 $\partial \mathcal{P}$ will again yield wasted space.

\section{The Matrix $\mathrm{C}_{\mathrm{op}}$}

We run a large while loop fixing each row in $C$. Once $c \in C$ is fixed, we then create the matrix $C_{o p} \subseteq C$ by again removing all rows in $C$ with common, non-zero elements of $c$. After the matrix $C_{o p}$ is created, we then apply Corollary 1 and remove rows in $C_{o p}$ that do not satisfy

$$
4\left(\left|a_{1}\right|+\left|a_{2}\right|+\left|a_{3}\right|+\left|a_{4}\right|\right) \leqslant\left|a_{4}\right|\left(\left|a_{4}\right|+1\right),
$$

where $a_{i}$ are the minimum squares on each of the edges and $\left|a_{i}\right|<\left|a_{i+1}\right|$. Finally, after including Corollary 1, we create the matrix (for display purposes) Frm which has rows $v, v_{o p}$, and $c$; we also display the matrix $C_{o p}$ to each corresponding Frm (count is used to count the number of frames, i.e., count adds 1 for every $c_{o p}$ generated). 


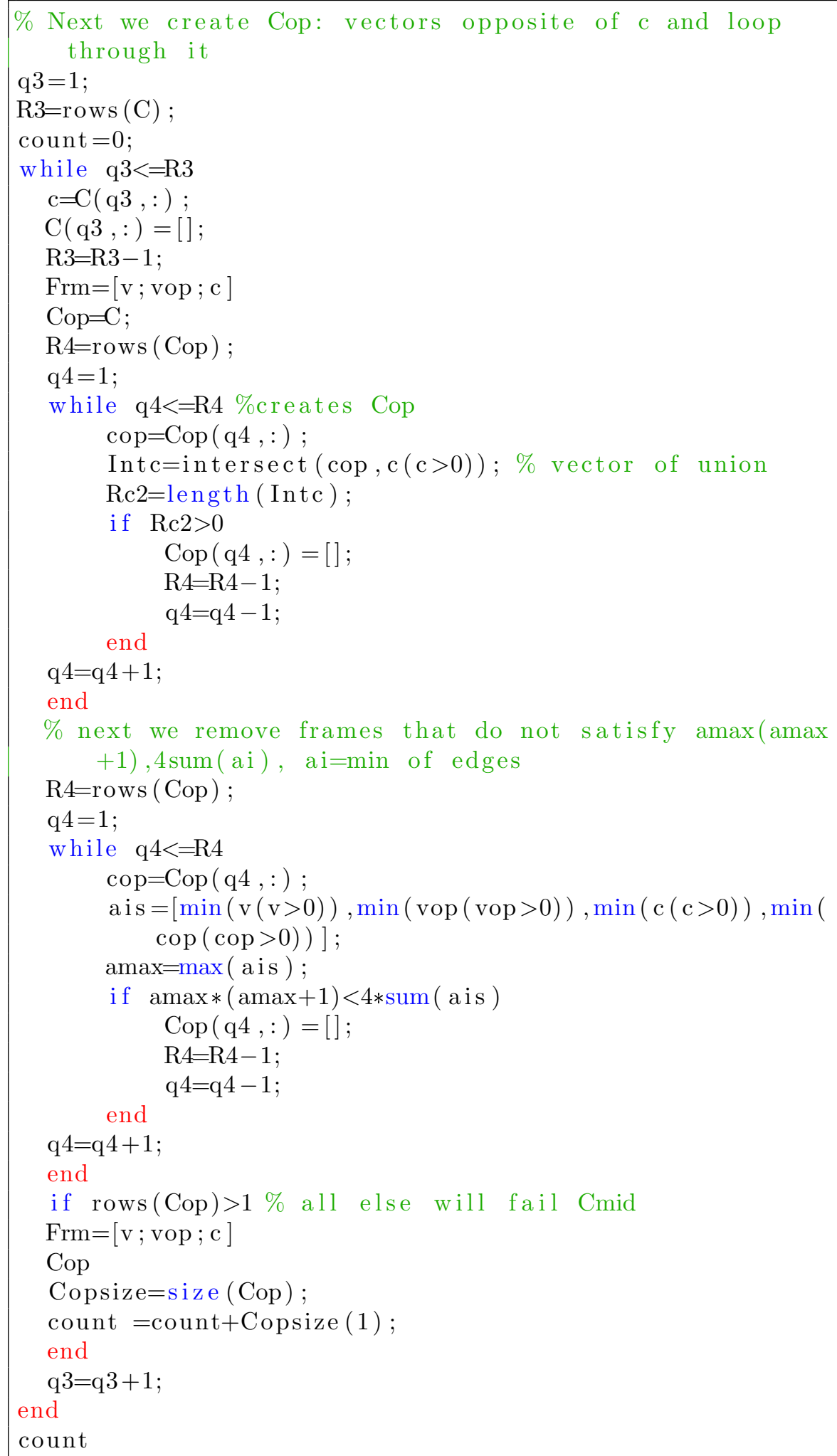




\section{Proof of Theorem 3}

Proof. Running our code from the preceding section with $v=\{7,8,14,18,23\}$ and $v_{o p}=\{9,10,11,12,13,15\}$, there are 56 frames to check. Each "Frm" is a matrix where the first and second rows are $v$ and $v_{o p}$, respectively. The third row is a different $c$ for each Frm. The matrix/vector following is Cop matrix, i.e., possibilities for the last edge.

The following Lemma will be important as it cuts the number of frames down from 56 to 9 .

Lemma 3. Given our fixed edges $v$ and $v_{o p}$, the 10 and 14-squares cannot be corner squares.

Proof. Suppose firstly the 10-square is a corner square. Consider the square that will be placed adjacent to the 10-square in the adjacent edge (i.e., $c$ or $\left.c_{o p}\right)$. Since the 7 -square is in $v$, then the 6 -square cannot be adjacent to the 10 -square. Further, squares $7-15$ have been placed in either $v$ or $v_{o p}$. Therefore, the next smallest square that can be placed adjacent to the 10-square in the adjacent edge is the 16-square. Therefore, the 9 -square is the only square that can be placed adjacent to the 10-square in $v_{o p}$. However, this guarantees wasted space over the 9 -square. Thus, the 10-square cannot be an edge.

Now, suppose the 14-square is a corner square. By the same reasoning, we know that the 16-square is next possible square that can be placed adjacent to the 14-square in the adjacent edge. This along with Theorem 1[(iii) implies we must have $\operatorname{adj}(7,8,14)$ on $v_{o p}$. Then the possible sets of squares to be placed on the 7 -square are $\operatorname{adj}(1,6), \operatorname{adj}(2,5), \operatorname{adj}(3,4), \operatorname{and} \operatorname{adj}(1,2,4)$. Consider when we have $\operatorname{adj}(7,14)$. $\operatorname{Adj}(1,2,4)$ is guaranteed wasted space adjacent to the 2-square for all permutations, and placing $\operatorname{adj}(1,6)$ on the 7 -square would leave a $1 \times 2$ (or longer) gap above the 6 -square. Therefore the set of squares on the 8 -square must sum to eight. Suppose $\operatorname{adj}(3,4)$ is placed on the 7 -square, then we must have $\operatorname{adj}(1,2,5)$ on the 8 -square. However, this guarantees wasted space over the 1 -square for all permutations. In a similar way, we rule out placing adj $(2,6)$ on the 8 -square. Suppose $\operatorname{adj}(2,5)$ is placed on the 7 -square. Then we must have $\operatorname{adj}(1,3,4)$ placed on the 8 -square. We must have $\operatorname{adj}(1,2)$ to avoid wasted space over either the 1 or 2 -square. This guarantees wasted space over the 1,2-flush.

This shows we cannot have adj(14,7). However, all cases were exhausted without the permutation of the 7 and 8-square taken into account except for $\operatorname{adj}(1,6)$ on the 7 -square; we only need to consider this case. Let ord $(14,8,7)$ be on $v_{o p}$, and place $\operatorname{adj}(1,6)$ on the 7 -square. Then we must have $\operatorname{adj}(1,8)$ to avoid wasted space over the 1-square. Then we need a set of squares that sum to nine to be placed on the 1,8-flush. The only sets $\operatorname{are} \operatorname{adj}(2,3,4)$ and $\operatorname{adj}(4,5)$. But $\operatorname{adj}(2,3,4)$ is ruled out by Lemma 2. Place adj $(4,5)$ on the 1,8 -flush. Then either permutation will leave wasted space over the 4 -square.

Therefore, we have exhausted all possibilities and so the 14 -square cannot be a corner square. 


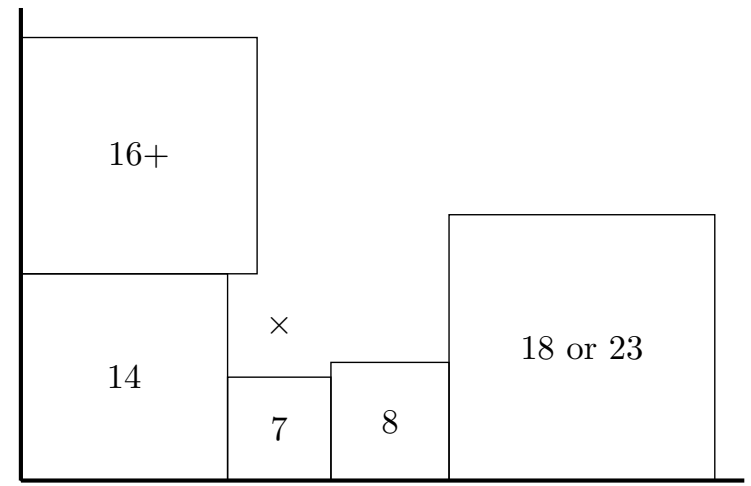

To account for this Lemma in the computer code, we add the following scripts before the code for generating Cop and Frm, and then run the code to generate the new set of possible frames.

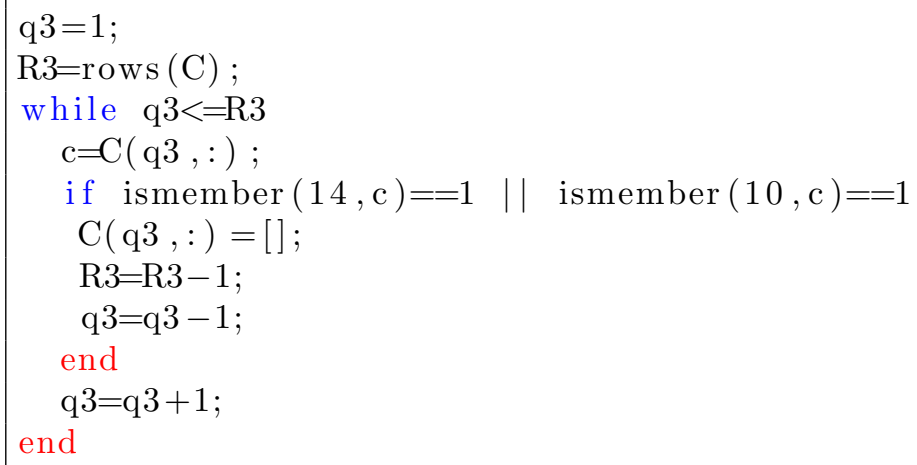

Note that this takes into consideration if a given $v, v_{o p}$, and $c$ yield zero $c_{o p}$ options, then these cases are ruled out immediately. This yields the following frames:

\begin{tabular}{|c|c|c|c|}
\hline Frm1 = & $\begin{array}{lllllll}0 & 0 & 7 & 8 & 14 & 18 & 23 \\
0 & 9 & 10 & 11 & 12 & 13 & 15 \\
0 & 0 & 0 & 11 & 16 & 20 & 23\end{array}$ & Cop $=$ & $\begin{array}{lllllll}0 & 0 & 0 & 12 & 18 & 19 & 21 \\
0 & 0 & 0 & 13 & 17 & 18 & 22\end{array}$ \\
\hline Frm2 $=$ & $\begin{array}{lllllll}0 & 0 & 7 & 8 & 14 & 18 & 23 \\
0 & 9 & 10 & 11 & 12 & 13 & 15 \\
0 & 0 & 0 & 11 & 16 & 20 & 23\end{array}$ & Cop $=$ & $\begin{array}{lllllll}0 & 0 & 0 & 12 & 16 & 18 & 24 \\
0 & 0 & 0 & 15 & 16 & 18 & 21\end{array}$ \\
\hline Frm3 = & $\begin{array}{lllllll}0 & 0 & 7 & 8 & 14 & 18 & 23 \\
0 & 9 & 10 & 11 & 12 & 13 & 15 \\
0 & 0 & 0 & 11 & 18 & 20 & 21\end{array}$ & Cop $=$ & $\begin{array}{lllllll}0 & 0 & 0 & 12 & 16 & 19 & 23\end{array}$ \\
\hline Frm4 $=$ & $\begin{array}{lllllll}0 & 0 & 7 & 8 & 14 & 18 & 23 \\
0 & 9 & 10 & 11 & 12 & 13 & 15 \\
0 & 0 & 0 & 12 & 16 & 19 & 23\end{array}$ & Cop $=$ & $\begin{array}{lllllll}0 & 0 & 0 & 11 & 17 & 18 & 24 \\
0 & 0 & 0 & 13 & 17 & 18 & 22 \\
0 & 0 & 0 & 15 & 17 & 18 & 20\end{array}$ \\
\hline Frm5 $=$ & $\begin{array}{lllllll}0 & 0 & 7 & 8 & 14 & 18 & 23 \\
0 & 9 & 10 & 11 & 12 & 13 & 15 \\
0 & 0 & 8 & 9 & 16 & 17 & 20\end{array}$ & Cop $=$ & $\begin{array}{lllllll}0 & 0 & 0 & 12 & 18 & 19 & 21\end{array}$ \\
\hline
\end{tabular}


Frames 1, 2, and 3 are all ruled out (for all choices of $c_{o p}$ ) as the 11-square is in the corner of $c$ and $v_{o p}$, but we must have $\operatorname{adj}(11,16)$ or $\operatorname{adj}(11,20)$ which yields wasted space to the right of the 11-square, for any $c_{o p}$.

For frame 4, we have the 12-square in the corner of $c$ and $v_{o p}$, where $\operatorname{adj}(12, \geqslant 16) \subset c$. We can see that the 9,10 , and 11-squares are the only possible squares that can be adjacent to the 12-square in $v_{o p}$. However, placing the 10 or 11 -square adjacent to the 12 -square leaves a $1 \times k_{1}$ or $2 \times k_{2}$ for $k_{1}, k_{2} \geqslant 4$. Therefore we must have adj $(9,12)$. Then the only tiling that can be placed above the 9 -square is placing the 3 -square adjacent to the 9 and 12 -squares, then placing adj $(1,2)$ on the 3 -square. Finally the 4 -square is the only remaining square that can be placed on the 9-square which makes the set of squares on the 9 -square sum to nine. However, this guarantees wasted space over the 2-square.

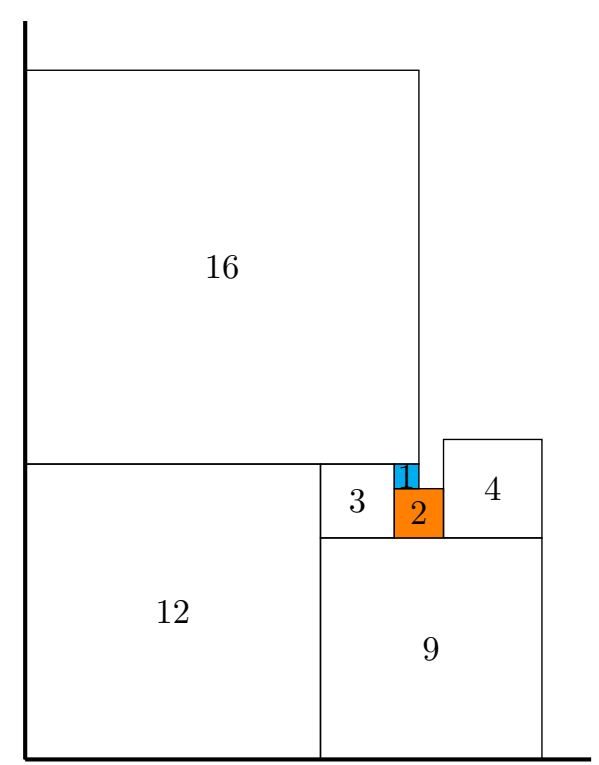

Finally, consider fame 5. Note the 8 and 9 -squares are a corner squares and the next smallest square to be adjacent to the 8-square in $c$ is the 16-square. That is, we must have $\operatorname{adj}(8, \geqslant 16)$, which contradicts Theorem[1|(iv), Therefore frame 5 is also ruled out.

Therefore, we have exhausted all possible frames for $v=\{7,8,14,18,23\}$ and $v_{o p}=\{9,10,11,12,13,15\}$. Thus these cannot both be edges opposite each other. 


\section{Near Misses}

Here is the MATLAB code to determine near-misses (Table 2.5).

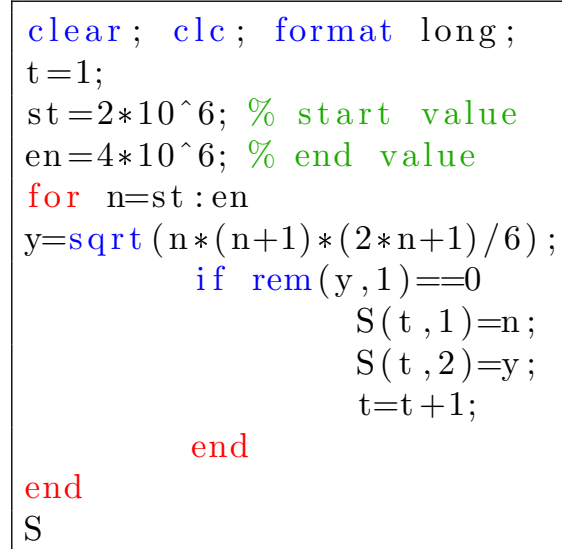

\section{References}

[1] Bitner, J., Reingold, E. "Backtrack Programming Techniques", Communications of the A.C.M., 18(11), 651-656, 1975.

[2] Gardner, M. "Mathematical Games: The problem of Mrs. Perkin's quilt and answers to last month's puzzles", Scientific American, 215(3), 264-272, 1966.

[3] Gardner, M. "The problem of Mrs. Perkin's quilt and other square-packing problems", Mathematical Carnival, New York: Alfred A. Knopf, 139-149, 1975 .

[4] Korf, R., Moffitt, M., Pollack, M. "Optimal rectangle packing", Ann Oper Res, 179, 261-295, 2010.

[5] Watson, G. "The problem of the square pyramid", Messenger of Mathematics, New Series, 48, 1-22, 1918. 